\title{
An effective strong-coupling theory of composite particles in UV-domain
}

\author{
She-Sheng Xue \\ ICRANet, \\ Piazzale della Repubblica 10, 10-65122, Pescara, Italy \\ Physics Department, Sapienza University of Rome, \\ Piazzale Aldo Moro 5, 00185 Roma, Italy \\ E-mail: xue@icra.it
}

ABSTRACT: We briefly review the effective field theory of massive composite particles, their gauge couplings and characteristic energy scale in the UV-domain of UV-stable fixed point of strong four-fermion coupling, then mainly focus the discussions on the decay channels of composite particles into the final states of the SM gauge bosons, leptons and quarks. We calculate the rates of composite bosons decaying into two gauge bosons $\gamma \gamma, \gamma Z^{0}, W^{+} W^{-}$, $Z^{0} Z^{0}$ and give the ratios of decay rates of different channels depending on gauge couplings only. It is shown that a composite fermion decays into an elementary fermion and a composite boson, the latter being an intermediate state decays into two gauge bosons, leading to a peculiar kinematics of final states of a quark (or a lepton) and two gauge bosons. These provide experimental implications of such an effective theory of composite particles beyond the SM. We also present some speculative discussions on the channels of composite fermions decaying into $W W, W Z$ and $Z Z$ two boson-tagged jets with quark jets, or to four-quark jets. Moreover, at the same energy scale of composite particles produced in high-energy experiments, composite particles are also produced by high-energy sterile neutrino (dark matter) collisions, their decays lead to excesses of cosmic ray particles in space and signals of SM particles in underground laboratories.

KeYwords: Effective Field Theories, Chiral Lagrangians

ARXIV EPRINT: 1601.06845 


\section{Contents}

1 Introduction 1

1.1 Weak-interacting four-fermion operators and symmetry-breaking phase 2

1.2 Strong-interacting four-fermion operators and gauge-symmetric phase 2

1.3 Article arrangement 3

2 Four-fermion operators beyond the SM 3

2.1 Regularization and quantum gravity 3

2.2 Einstein-Cartan theory with SM gauge symmetries and fermion content 4

2.3 Fermion-family symmetry and mass eigenstate 5

$\begin{array}{lll}2.4 & \text { SM gauge-symmetric four-fermion operators } & 7\end{array}$

$\begin{array}{lll}2.5 & \text { Four-fermion operators of quark-lepton interactions } & 7\end{array}$

3 IR-stable and UV-stable fixed points and their scaling regions $\quad 8$

3.1 Symmetry-breaking phase and the IR-domain of the IR-stable fixed point 8

3.1.1 Resolution to the $t \bar{t}$-condensate model 9

3.1.2 Experimental indications of tightly-bound composite Higgs boson? 11

3.1.3 Yukawa couplings and FCNC's in IR-domain 12

$\begin{array}{lll}3.2 & \text { Gauge-symmetric phase of strong four-fermion coupling } & 13\end{array}$

$\begin{array}{ll}\text { 3.3 Strong critical coupling and the UV-stable fixed point } & 14\end{array}$

$\begin{array}{ll}\text { 3.3.1 Strong critical coupling } & 14\end{array}$

$\begin{array}{ll}\text { 3.3.2 UV-stable fixed point } & 15\end{array}$

$\begin{array}{lll}3.4 & \text { The UV-domain of UV-stable fixed point } & 16\end{array}$

$\begin{array}{lll}3.4 .1 & \text { Energy threshold of composite particles } & 16\end{array}$

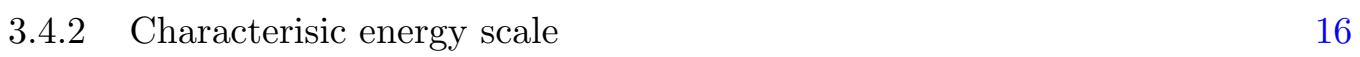

3.5 Relevant and irrelevant operators in IR- and UV-domains 17

4 Composite particles and effective Lagrangian in UV-domain 19

$\begin{array}{lll}4.1 & \text { Composite particles in UV-domain } & 19\end{array}$

$\begin{array}{lll}4.1 .1 & \text { Quark sector } & 19\end{array}$

$\begin{array}{lll}\text { 4.1.2 Lepton sector } & 20\end{array}$

$\begin{array}{lll}\text { 4.1.3 } & \text { Quark-lepton sector } & 20\end{array}$

4.1.4 Discussions and three-family replication 21

4.2 Effective Lagrangian of composite particles in UV-domain 21

4.2.1 SM effective Lagrangian of elementary particles in IR-domain 21

4.2.2 Effective Lagrangian of composite particles in UV-domain 22

4.2.3 Some discussions on FCNC and anomalies in UV-domain 24 
5 Composite pesudo scalar bosons decay $\quad 26$

$\begin{array}{lll}5.1 & \text { Low-energy QCD pions and their decays } & 26\end{array}$

$\begin{array}{lll}5.2 & \text { Scalar and pesudo scalar composite bosons } & 27\end{array}$

5.3 Composite particle $\Pi^{0}$ decay into two SM gauge bosons 28

$\begin{array}{lll}\text { 5.3.1 } & \Pi^{0} \text { decay into two photons } & 28\end{array}$

5.3.2 $\Pi^{0}$ decay into $\gamma+Z^{0}, W^{+}+W^{-}$and $Z^{0}+Z^{0} \quad 30$

5.4 Ratios of different channels of $\Pi^{0}$ decay into two SM gauge bosons 31

$5.5 \Pi^{ \pm}$and other composite boson decays 34

6 Composite fermion decay and annihilation channels 34

6.1 Composite fermions decay 34

6.1.1 Decay into two gauge bosons and a quark 34

6.1.2 Decay into two gauge bosons and a lepton 35

6.1.3 Other channels of composite fermion decay 37

$\begin{array}{lll}6.2 & \text { Annihilation of two composite fermions } & 37\end{array}$

$\begin{array}{lll}7 & \text { Some speculative considerations for experiments } & 39\end{array}$

$\begin{array}{lll}7.1 & \text { Some speculative considerations for LHC experiments } & 39\end{array}$

7.2 Sterile neutrinos interacting with SM particles at high energies 40

$\begin{array}{lll}7.3 & \text { Further studies } & 41\end{array}$

\section{Introduction}

The parity-violating (chiral) gauge symmetries and spontaneous/explicit breaking of these symmetries for the hierarchy of fermion masses have been at the center of a conceptual elaboration that has played a major role in donating to mankind the beauty of the SM for particle physics. The Nambu-Jona-Lasinio (NJL) model [1] of dimension-6 four-fermion operators at high energies and its effective counterpart, the phenomenological model [27] of the elementary Higgs boson and its Yukawa-coupling to fermions at low energies, provide an elegant and simple description for the electroweak symmetry breaking and intermediate gauge boson masses. After a great experimental effort for many years, using data recorded at $\sqrt{s}=7,8 \mathrm{TeV}$ experiments of $p p$ collision at the Large Hadron Collider (LHC), the ATLAS [8] and CMS [9] collaborations have shown the first observations of a $125 \mathrm{GeV}$ scalar particle in the search for the SM Higgs boson. This far-reaching result begins to shed light on this most elusive and fascinating arena of fundamental particle physics. Recently, in the Run-2 of the upgraded LHC, the preliminary results on $\sqrt{s}=13 \mathrm{TeV}$ $p p$ collision data are prepared by ATLAS [10] and CMS [11] to search for new (beyond the SM) resonant and/or nonresonant phenomena that might manifest themselves in the high-energy regime with final states of diboson, dilepton, dijet and so forth. The diphoton channel preliminarily showed the trace of a new resonance at the diphoton invariant mass $\mathcal{M}_{\gamma \gamma}$, which however has not been further confirmed by increasing the integrated luminosity and energy of $p p$ collisions. 


\subsection{Weak-interacting four-fermion operators and symmetry-breaking phase}

The dynamics of new physics at high energies may be represented by an effective theory of high-dimensional operators of fermion fields, e.g., dimension- 6 four-fermion operators, preserving at least the SM gauge symmetries. The strong technicolor dynamics of extended gauge theories at the $\mathrm{TeV}$ scale was invoked [22-27] to have a natural scheme incorporating the relevant four-fermion operator $G\left(\bar{\psi}_{L}^{i a} t_{R a}\right)\left(\bar{t}_{R}^{b} \psi_{L i b}\right)$ of the $\langle\bar{t} t\rangle$-condensate model [28], to generate the top-quark mass via spontaneous symmetry breaking (SSB). On the other hand, these relevant operators can be constructed on the basis of phenomenology of the SM at low-energies. In 1989, several authors [28-33] suggested that the SM symmetry breakdown could be a dynamical mechanism of the NJL type that intimately involves the top quark at the high-energy scale $\Lambda$. Since then, many models based on this idea have been studied $[34,35]$. In the scenes of renormalization group (scaling) invariance and resultant renormalization-group (RG) equations, there is a scaling region (IR-domain) of the infrared (IR) stable fixed point of four-fermion operators, the low-energy SM physics was supposed to be achieved by the RG equations in the IR-domain with the electroweak scale $v \approx$ $239.5 \mathrm{GeV}[25-28,33]$. It is in this IR-domain, we recently present the detailed study of hierarchy mass spectrum of SM fermions: from top quark to electron neutrino [12, 13], on the basis of effective four-fermion operators of Einstein-Cartan type, as will be shown in section 2 .

\subsection{Strong-interacting four-fermion operators and gauge-symmetric phase}

In addition to the IR-domain of weak-coupling four-fermion operators in the SSB phase where the low-energy SM is realized, we find a scaling region (UV-domain) of the ultraviolet (UV) stable fixed point of strong-coupling four-fermion operators in the gauge-symmetric phase. In this UV-domain at high energies, it realizes an effective theory of composite bosons and fermions composed by SM elementary fermions, these composite particles and their interactions preserving the SM gauge symmetries [14-20]. This is the issue that we would like to focus in this article.

In refs. [20,21], we have already presented discussions on the possible decay channels of composite bosons and fermions into final states of jets and leptons in the framework of effective four-fermion operators in high energies. In this article, we focus on the discussions on the possible channels of composite bosons decaying into two SM gauge bosons, and composite fermions decaying into two SM gauge bosons (diboson) and a quark/lepton, as well as their experimental implications. It is preliminarily shown that the diphoton channel of composite bosons decay should have the largest branching ratio, however other diboson channels should also contribute to the invariant mass $\mathcal{M}$ of possible composite-boson resonance. Instead, composite fermions decay into a composite boson and a quark/lepton, the former is an intermediate state, which then decays into two bosons, i.e., a quark-diboson or lepton-diboson channel. These final states and their kinematic relations of such a quarkdiboson or lepton-diboson channel are peculiar. This provides a possibility/criterion to verify the effective theory of composite particles in high-energy experiments. 


\subsection{Article arrangement}

In section 2, we present the SM gauge-symmetric operators of four-fermion interactions of Einstein-Cardan type and the brief discussion of their possible origin. Some explanations and clarifications of the low-energy IR-domain for the SM and the high-energy UV-domain for an effective field theory of composite particles are given in section 3. After showing the composite particle spectrum and effective Lagrangian in sections 4 in the framework of SM fermion content and gauge symmetries, we discuss in sections 5 and 6.1 the main results that the composite boson and fermion decay into SM gauge bosons and/or elementary fermions in connection with high-energy experiments. In the final section, we present some discussions and speculations on the possible channels of composite particles interacting with SM particles, that could be relevant to some experiments at the energy scale of composite particles.

\section{Four-fermion operators beyond the SM}

In order for a self-contained and self-consistent article, as well as for readers' convenience, we include this section of describing effective four-fermion operators at the cutoff $\Lambda$ that was similarly presented in ref. [13] for discussing the hierarchy spectrum of SM fermions in the IR-domain. It is also necessary to present these effective four-fermion operators at the cutoff $\Lambda$ to clarify its induced Lagrangian of the composite particle spectra and interactions in the UV-domain.

\subsection{Regularization and quantum gravity}

Up to now the theoretical and experimental studies tell us the chiral gauge-field interactions to fermions in the lepton-quark family that is replicated three times and mixed. The spontaneous breaking of these chiral gauge symmetries and generating of fermion masses are made by the Higgs field sector. In the IR-fixed-point domain of weak four-fermion coupling or equivalently weak Yukawa coupling, the SM Lagrangian with all relevant operators (parametrizations) is realized and behaves an effective and renormalizable field theory in low energies. To achieve these SM relevant operators, a finite field theory of chiral-gauge interactions should be well-defined by including the quantum gravity that naturally provides a space-time regularization (UV cutoff). As an example, the finite superstring theory is proposed by postulating that instead of a simple space-time point, the fundamental spacetime "constituents" is a space-time "string". The Planck scale is a plausible cut-off, at which all principle and symmetries are fully respected by gauge fields and particle spectra, fermions and bosons.

In this article, we do not discuss how a fundamental theory at the Planck scale induces high-dimensional operators. Instead, as a postulation or motivation, we argue the presence of at least four-fermion operators beyond the SM from the following point view. A well-defined quantum field theory for the SM Lagrangian requires a natural regularization (UV cutoff $\Lambda$ ) fully preserving the SM chiral-gauge symmetry. The quantum gravity naturally provides a such regularization of discrete space-time with the minimal length $\tilde{a} \approx$ 
$1.2 a_{\mathrm{pl}}$ [36-39], where the Planck length $a_{\mathrm{pl}} \sim 10^{-33} \mathrm{~cm}$ and scale $\Lambda_{\mathrm{pl}}=\pi / a_{\mathrm{pl}} \sim 10^{19} \mathrm{GeV}$. However, the no-go theorem [40-42] tells us that there is no any consistent way to regularize the SM bilinear fermion Lagrangian to exactly preserve the SM chiral-gauge symmetries, which must be explicitly broken at the scale of fundamental space-time cutoff $\tilde{a}$. This implies that the natural quantum-gravity regularization for the SM should lead us to consider at least dimension- 6 four-fermion operators originated from quantum gravity effects at short distances. ${ }^{1}$ As a model, we adopt the four-fermion operators of the torsion-free Einstein-Cartan Lagrangian within the framework of the SM fermion content and gauge symmetries. We stress that a fundamental theory at the UV cutoff is still unknown.

\subsection{Einstein-Cartan theory with SM gauge symmetries and fermion content}

The Lagrangian of torsion-free Einstein-Cartan (EC) theory reads,

$$
\mathcal{L}_{E C}(e, \omega, \psi)=\mathcal{L}_{E C}(e, \omega)+\bar{\psi} e^{\mu} \mathcal{D}_{\mu} \psi+G J^{d} J_{d}
$$

where the gravitational Lagrangian $\mathcal{L}_{E C}=\mathcal{L}_{E C}(e, \omega)$, tetrad field $e_{\mu}(x)=e_{\mu}{ }^{a}(x) \gamma_{a}$, spinconnection field $\omega_{\mu}(x)=\omega_{\mu}^{a b}(x) \sigma_{a b}$, the covariant derivative $\mathcal{D}_{\mu}=\partial_{\mu}-i g \omega_{\mu}$ and the axial current $J^{d}=\bar{\psi} \gamma^{d} \gamma^{5} \psi$ of massless fermion fields. The four-fermion coupling $G$ relates to the gravitation-fermion gauge coupling $g$ and fundamental space-time cutoff $\tilde{a}$.

In the context of the SM one-family fermion content and gauge symmetries, we consider massless, two-component, left- and right-handed Weyl fermions $\psi_{L}^{f}$ (doublets) and $\psi_{R}^{f}$ (singlets) carrying the quantum numbers of the SM $S U_{L}(2) \times U_{Y}(1)$ chiral gauge symmetries, where " $f$ " is the fermion-family index, as well as three right-handed Weyl sterile neutrinos $\nu_{R}^{f}$ and their left-handed conjugated fields $\nu_{R}^{f c}=i \gamma_{2}\left(\nu_{R}\right)^{*}$, which do not carry any quantum number of SM gauge symmetries. Analogously to the EC theory (2.1), we obtain a torsion-free, diffeomorphism and local gauge-invariant Lagrangian

$$
\begin{aligned}
\mathcal{L}= & \mathcal{L}_{E C}(e, \omega)+\sum_{f} \bar{\psi}_{L, R}^{f} e^{\mu} \mathcal{D}_{\mu} \psi_{L, R}^{f}+\sum_{f} \bar{\nu}_{R}^{f c} e^{\mu} \mathcal{D}_{\mu} \nu_{R}^{f c} \\
& +G\left(J_{L}^{\mu} J_{L, \mu}+J_{R}^{\mu} J_{R, \mu}+2 J_{L}^{\mu} J_{R, \mu}\right) \\
& +G\left(j_{L}^{\mu} j_{L, \mu}+2 J_{L}^{\mu} j_{L, \mu}+2 J_{R}^{\mu} j_{L, \mu}\right)
\end{aligned}
$$

where the SM gauge fields $A_{\mu}$ are present in the co-variant derivative $\mathcal{D}_{\mu}$ to preserve the SM gauge symmetries, and axial currents read

$$
J_{L, R}^{\mu} \equiv \sum_{f} \bar{\psi}_{L, R}^{f} \gamma^{\mu} \gamma^{5} \psi_{L, R}^{f}, \quad j_{L}^{\mu} \equiv \sum_{f} \bar{\nu}_{R}^{f c} \gamma^{\mu} \gamma^{5} \nu_{R}^{f c}
$$

The four-fermion coupling $G$ is unique for all four-fermion operators and high-dimensional fermion operators $(d>6)$ are neglected.

\footnotetext{
${ }^{1}$ In the regularized and quantized EC theory [36-39] with a basic space-time cutoff, in addition to dimension-6 four-fermion operators, there are high-dimensional fermion operators $(d>6)$, e.g., $\partial_{\sigma} J^{\mu} \partial^{\sigma} J_{\mu}$, which are suppressed at least by $\mathcal{O}\left(\tilde{a}^{4}\right)$.
} 
By using the Fierz theorem $[43,44]$, the dimension- 6 four-fermion operators in eq. (2.2) can be written as [21]

$$
\begin{aligned}
& +(G / 2)\left(J_{L}^{\mu} J_{L, \mu}+J_{R}^{\mu} J_{R, \mu}+j_{L}^{\mu} j_{L, \mu}+2 J_{L}^{\mu} j_{L, \mu}\right) \\
& -G \sum_{f f^{\prime}}\left(\bar{\psi}_{L}^{f} \psi_{R}^{f^{\prime}} \bar{\psi}_{R}^{f^{\prime}} \psi_{L}^{f}+\bar{\nu}_{R}^{f c} \psi_{R}^{f^{\prime}} \bar{\psi}_{R}^{f^{\prime}} \nu_{R}^{f c}\right)
\end{aligned}
$$

which preserve the SM gauge symmetries. Equations (2.4) and (2.5) represent repulsive and attractive operators respectively. The former (2.4) are suppressed by the cutoff $\mathcal{O}\left(\Lambda^{-2}\right)$, and cannot become relevant and renormalizable operators of effective dimension- 4 . Thus the torsion-free EC theory with the attractive four-fermion operators reads,

$$
\begin{aligned}
\mathcal{L}= & \mathcal{L}_{E C}+\sum_{f} \bar{\psi}_{L, R}^{f} e^{\mu} \mathcal{D}_{\mu} \psi_{L, R}^{f}+\sum_{f} \bar{\nu}_{R}^{f c} e^{\mu} \mathcal{D}_{\mu} \nu_{R}^{f c} \\
& -G \sum_{f f^{\prime}}\left(\bar{\psi}_{L}^{f} \psi_{R}^{f^{\prime}} \bar{\psi}_{R}^{f^{\prime}} \psi_{L}^{f}+\bar{\nu}_{R}^{f c} \psi_{R}^{f^{\prime}} \bar{\psi}_{R}^{f^{\prime}} \nu_{R}^{f c}\right)+\text { h.c. },
\end{aligned}
$$

where the two component Weyl fermions $\psi_{L}^{f}$ and $\psi_{R}^{f}$ respectively are the $S U_{L}(2) \times U_{Y}(1)$ gauged doublets and singlets of the SM. For the sake of compact notations, $\psi_{R}^{f}$ are also used to represent $\nu_{R}^{f}$, which have no any SM quantum numbers. All fermions are massless, they are four-component Dirac fermions $\psi^{f}=\left(\psi_{L}^{f}+\psi_{R}^{f}\right)$, two-component left-handed Weyl neutrinos $\nu_{L}^{f}$ and four-component sterile Majorana neutrinos $\nu_{M}^{f}=\left(\nu_{R}^{f c}+\nu_{R}^{f}\right)$ whose kinetic terms read

$$
\bar{\nu}_{L}^{f} e^{\mu} \mathcal{D}_{\mu} \nu_{L}^{f}, \quad \bar{\nu}_{M}^{f} e^{\mu} \mathcal{D}_{\mu} \nu_{M}^{f}=\bar{\nu}_{R}^{f} e^{\mu} \mathcal{D}_{\mu} \nu_{R}^{f}+\bar{\nu}_{R}^{f c} e^{\mu} \mathcal{D}_{\mu} \nu_{R}^{f c} .
$$

In eq. (2.6), $f$ and $f^{\prime}\left(f, f^{\prime}=1,2,3\right)$ are fermion-family indexes summed over respectively for three lepton families (charge $q=0,-1)$ and three quark families $(q=2 / 3,-1 / 3)$. Equation (2.6) preserves not only the SM gauge symmetries and global fermion-family symmetries, but also the global symmetries for fermion-number conservations. We adopt the effective four-fermion operators (2.6) in the context of a well-defined quantum field theory at the high-energy scale $\Lambda$.

\subsection{Fermion-family symmetry and mass eigenstate}

As argued in the introduction section 2.1, the origin of effective four-fermion operators in eqs. (2.1)-(2.6) is due to the quantum gravity that couples to all fermion fields and provides a natural regularization for chiral gauge field theories, like the SM, at the UV cutoff $\Lambda$. Therefore, there is no any reason to assume different four-fermion coupling $G$ 's for different fermions that equally couple to the gravitational field in the torson-free Einstein-Cartan theory (2.1).

It should be further clarified that in the effective Lagrangian (2.6) at the cutoff $\Lambda$, (i) the massless SM fermion fields are interacting gauge eigenstates of the SM gauge symmetries $S U_{c}(3) \times S U_{L}(2) \times U_{Y}(1)$; (ii) due to the unique four-fermion coupling $G$, there are exact global fermion-family $U_{L}(3) \times U_{R}(3)$ chiral symmetries, i.e., flavor or horizon symmetries with respect to different charges $q=0,-1,2 / 3,-1 / 3$ of fermions in different families. 
In order to study the gauge-boson-fermion and four-fermion interactions in terms of fermion mass-energy spectra and currents, measured as physical final states, we adopt the energy-mass eigenstates of fermions in the following discussions of entire article. The unitary chiral transformations $\mathcal{U}_{L} \in U_{L}(3)$ and $\mathcal{U}_{R} \in U_{R}(3)$, where $\mathcal{U}_{L}$ and $\mathcal{U}_{R}$ are related by a unitary matrix $\mathcal{V}$, can be performed from gauge eigenstates to mass eigenstates (upand down-quark sectors as example):

$$
\psi_{L}^{u} \rightarrow \mathcal{U}_{L}^{u} \psi_{L}^{u}, \quad \psi_{R}^{u} \rightarrow \mathcal{U}_{R}^{u} \psi_{R}^{u} ; \quad \mathcal{U}_{L, R}^{u} \in U_{L, R}^{u}(3)
$$

and

$$
\psi_{L}^{d} \rightarrow \mathcal{U}_{L}^{d} \psi_{L}^{d}, \quad \psi_{R}^{d} \rightarrow \mathcal{U}_{R}^{d} \psi_{R}^{d} ; \quad \mathcal{U}_{L, R}^{d} \in U_{L, R}^{d}(3),
$$

so that in eq. (2.6) the fermion-family indexes $f=f^{\prime}$, i.e., $\delta_{f f^{\prime}}$ respectively for the $u$ quark sector and the $d$-quark sector. As a result, all quark fields are mass eigenstates, the four-fermion operators (2.6) are "diagonal" only for each quark family without fermionfamily mixing,

$$
-G \sum_{f=1,2,3}\left[\bar{\psi}_{L}^{f} \psi_{R}^{f} \bar{\psi}_{R}^{f} \psi_{L}^{f}\right]_{2 / 3}^{\mathrm{up}}-G \sum_{f=1,2,3}\left[\bar{\psi}_{L}^{f} \psi_{R}^{f} \bar{\psi}_{R}^{f} \psi_{L}^{f}\right]_{-1 / 3}^{\text {down }}
$$

In the following, we adopt this representation and our notations will be only for the first SM family, however, they are the same for the second and third families.

In this representation, bilinear fermion-mass operators $\left\langle\psi_{R}^{f} \bar{\psi}_{L}^{f}\right\rangle+$ h.c., i.e., quark-mass matrices are diagonalized in the fermion-family space by the biunitary transformations

$$
\begin{aligned}
& M^{u} \Rightarrow M_{\text {diag }}^{u}=\left(m_{1}^{u}, m_{2}^{c}, m_{3}^{t}\right)=\mathcal{U}_{L}^{u \dagger} M^{u} \mathcal{U}_{R}^{u}, \\
& M^{d} \Rightarrow M_{\text {diag }}^{d}=\left(m_{1}^{d}, m_{2}^{s}, m_{3}^{b}\right)=\mathcal{U}_{L}^{d \dagger} M^{d} \mathcal{U}_{R}^{d},
\end{aligned}
$$

where all quark masses (eigenvalues) are positive, $\mathcal{U}_{L}$ and $\mathcal{U}_{R}$ are related by

$$
\mathcal{U}_{L}^{u, d}=\mathcal{V}^{u, d} \mathcal{U}_{R}^{u, d}
$$

and $\mathcal{V}^{u, d}$ is a unitary matrix, see for example [45, 46]. Using unitary matrices $\mathcal{U}_{L, R}^{u}(2.8)$ and $\mathcal{U}_{L, R}^{d}(2.9)$, up to a diagonal phase matrix we define the unitary quark-family mixing matrices,

$$
\begin{array}{ll}
\mathcal{U}_{L}^{u \dagger} \mathcal{U}_{L}^{d}, & \mathcal{U}_{L}^{u \dagger} \mathcal{U}_{R}^{d}, \\
\mathcal{U}_{R}^{u \dagger} \mathcal{U}_{L}^{d}, & \mathcal{U}_{R}^{u \dagger} \mathcal{U}_{R}^{d}
\end{array}
$$

where the first element is the CKM matrix $U=U_{L}^{q} \equiv \mathcal{U}_{L}^{u \dagger} \mathcal{U}_{L}^{d}$. The similar discussions for the lepton sector can be found in ref. [13].

In the IR effective theory in the IR-domain, the nonzero and different expectational values of fermion-mass operators $\left\langle\psi_{R}^{f} \bar{\psi}_{L}^{f}\right\rangle \propto m^{f} \neq 0$ (2.11) and fermion-family hierarchy $m^{f} \neq m^{f^{\prime}}$ can be developed due to both spontaneous-symmetry and explicit-symmetry breaking of chiral symmetries [12]. In this case, apart from the breaking of the SM chiral gauge symmetries, fermion-family (flavor) $U_{L}(3) \times U_{R}(3)$ symmetries are broken to the 
$\mathrm{U}(1)$ symmetry for each fermion family, i.e., $U_{1}(1) \times U_{2}(1) \times U_{3}(1)$ for the four-fermion operators (2.10), two-fermion operators (2.11) and (2.12). However, the mass eigenstates of the SM elementary fermions are different from their chiral-gauge eigenstates, i.e., the interaction vertexes of the chiral-gauge-boson $W^{ \pm}$and massive fermions are not diagonal in the fermion-family space based on the mass eigenstates of fermions, as can be seen in the kinetic terms of the effective Lagrangian (2.6).

In the UV effective theory in the UV-domain, instead, the chiral and flavor symmetries are preserved for $m^{f}=0$, indicating that gauge and mass eigenstates of composite particles are the same. In sections 3.1.3 and 4.2.3, we will come back to the discussions of the flavorchanging-neutral-current (FCNC) processes, considering the chiral- and flavor-symmetries breaking (preserving) of IR (UV) effective theory in the IR (UV) domain respectively.

\subsection{SM gauge-symmetric four-fermion operators}

Using eq. (2.10) and we explicitly show SM gauge symmetric four-fermion operators. In the quark sector, the four-fermion operators are

$$
G\left[\left(\bar{\psi}_{L}^{i a} t_{R a}\right)\left(\bar{t}_{R}^{b} \psi_{L i b}\right)+\left(\bar{\psi}_{L}^{i a} b_{R a}\right)\left(\bar{b}_{R}^{b} \psi_{L i b}\right)\right]+\text { "terms", }
$$

where $a, b$ and $i, j$ are the color and flavor indexes of the top and bottom quarks, the quark $S U_{L}(2)$ doublet $\psi_{L}^{i a}=\left(t_{L}^{a}, b_{L}^{a}\right)$ and singlet $\psi_{R}^{a}=t_{R}^{a}, b_{R}^{a}$ are the eigenstates of electroweak interaction. The first and second terms in eq. (2.15) are respectively the four-fermion operators of top-quark channel [28] and bottom-quark channel, whereas "terms" stands for the first and second quark families that can be obtained by substituting $t \rightarrow u, c$ and $b \rightarrow d, s[20,50,51]$.

In the lepton sector with three right-handed sterile neutrinos $\nu_{R}^{\ell}(\ell=e, \mu, \tau)$, the four-fermion operators in terms of gauge eigenstates are,

$$
G\left[\left(\bar{\ell}_{L}^{i} \ell_{R}\right)\left(\bar{\ell}_{R} \ell_{L i}\right)+\left(\bar{\ell}_{L}^{i} \nu_{R}^{\ell}\right)\left(\bar{\nu}_{R}^{\ell} \ell_{L i}\right)+\left(\bar{\nu}_{R}^{\ell c} \nu_{R}^{\ell}\right)\left(\bar{\nu}_{R}^{\ell} \nu_{R}^{\ell c}\right)\right]
$$

preserving all SM gauge symmetries, where the lepton $S U_{L}(2) \operatorname{doublets} \ell_{L}^{i}=\left(\nu_{L}^{\ell}, \ell_{L}\right)$, singlets $\ell_{R}$ and the conjugate fields of sterile neutrinos $\nu_{R}^{\ell c}=i \gamma_{2}\left(\nu_{R}^{\ell}\right)^{*}$. Coming from the second term in eq. (2.6), the last term in eq. (2.16) preserves the symmetry $U_{\text {lepton }}(1)$ for the lepton-number conservation, although $\left(\bar{\nu}_{R}^{\ell} \nu_{R}^{\ell c}\right)$ violates the lepton number of family " $\ell$ " by two units.

Similarly, from the second term in eq. (2.6) there are following four-fermion operators

$$
G\left[\left(\bar{\nu}_{R}^{\ell c} \ell_{R}\right)\left(\bar{\ell}_{R} \nu_{R}^{\ell c}\right)+\left(\bar{\nu}_{R}^{\ell c} u_{a, R}^{\ell}\right)\left(\bar{u}_{a, R}^{\ell} \nu_{R}^{\ell c}\right)+\left(\bar{\nu}_{R}^{\ell c} d_{a, R}^{\ell}\right)\left(\bar{d}_{a, R}^{\ell} \nu_{R}^{\ell c}\right)\right],
$$

where quark fields $u_{a, R}^{\ell}=(u, c, t)_{a, R}$ and $d_{a, R}^{\ell}=(d, s, b)_{a, R}$.

\subsection{Four-fermion operators of quark-lepton interactions}

Although the four-fermion operators in eq. (2.6) do not have quark-lepton interactions, we consider the following SM gauge-symmetric four-fermion operators that contain quarklepton interactions $[12,13,47]$,

$$
G\left[\left(\bar{\ell}_{L}^{i} e_{R}\right)\left(\bar{d}_{R}^{a} \psi_{L i a}\right)+\left(\bar{\ell}_{L}^{i} \nu_{R}^{e}\right)\left(\bar{u}_{R}^{a} \psi_{L i a}\right)\right]+(\cdots),
$$


where $\ell_{L}^{i}=\left(\nu_{L}^{e}, e_{L}\right)$ and $\psi_{L i a}=\left(u_{L a}, d_{L a}\right)$ for the first family. The $(\cdots)$ represents for the second and third families with substitutions: $e \rightarrow \mu, \tau, \nu^{e} \rightarrow \nu^{\mu}, \nu^{\tau}$, and $u \rightarrow c, t$ and $d \rightarrow s, b$. The four-fermion operators (2.18) of quark-lepton interactions are not included in eq. (2.6), since leptons and quarks are in separated representations of SM gauge groups. They should be expected in the framework of Einstein-Cartan theory and $\mathrm{SO}(10)$ unification theory $[48,49]$.

\section{IR-stable and UV-stable fixed points and their scaling regions}

Apart from what is possible new physics at the UV scale $\Lambda$ explaining the origin of these effective four-fermion operators, it is essential and necessary to study: (i) the phase diagram in the space of these effective four-fermion operator couplings; (ii) which dynamics of these operators undergo in terms of their couplings as functions of running energy scale $\mu$; (iii) associating to these dynamics where infrared (IR) and/or ultraviolet (UV) stable fixed point of physical couplings locates; (iv) in the IR and/or UV domains (scaling regions) of these stable fixed points, which operators become physically relevant and renormalizable following RG equations (scaling laws), and other irrelevant operators are suppressed by the cutoff at least $\mathcal{O}\left(\Lambda^{-2}\right)$.

\subsection{Symmetry-breaking phase and the IR-domain of the IR-stable fixed point}

In the NJL symmetry-breaking phase of weak coupling $G>G_{c}{ }^{2}$ where $G_{c}$ is the weak critical coupling of the NJL dynamics, the low-energy SM physics was supposed to be achieved in the IR-domain $\left(G \gtrsim G_{c}\right)$ of IR-stable fixed point [25-28, 33]. Bardeen, Hill and Lindner (BHL) proposed the effective Lagrangian of the $t \bar{t}$-condensate model [28],

$$
\begin{aligned}
L= & L_{\text {kinetic }}+g_{t 0}\left(\bar{\psi}_{L} t_{R} H+\text { h.c. }\right)+\Delta L_{\text {gauge }} \\
& +Z_{H}\left|D_{\mu} H\right|^{2}-m_{0}^{2} H^{\dagger} H-\frac{\lambda_{0}}{2}\left(H^{\dagger} H\right)^{2},
\end{aligned}
$$

with a massive composite Higgs boson $H=(\bar{t} t)$ and massless Goldstone bosons $\left(\bar{t} \gamma_{5} t\right)$ and $\left(\bar{t} \gamma_{5} b\right)$, which are the longitudinal modes of massive intermediate gauge bosons. The composite Higgs' bare form-factor (wave-function renormalization) $Z_{H}$ and masss $m_{0} H$, as well as its Yukawa $g_{t 0}$ and quartic $\lambda_{0}$ couplings are defined at an intermediate scale $\mathcal{E}(v<\mathcal{E}<\Lambda)$, that will be clear below (3.12). Renormalized quantities, like Yukawa coupling $\bar{g}_{t}\left(\mu^{2}\right)$ and quartic couplings $\bar{\lambda}\left(\mu^{2}\right)$ are defined at the running energy scale $\mu$ and satisfy the RG equations in the IR-domain,

$$
\begin{aligned}
16 \pi^{2} \frac{d \bar{g}_{t}}{d t} & =\left(\frac{9}{2} \bar{g}_{t}^{2}-8 \bar{g}_{3}^{2}-\frac{9}{4} \bar{g}_{2}^{2}-\frac{17}{12} \bar{g}_{1}^{2}\right) \bar{g}_{t}, \\
16 \pi^{2} \frac{d \bar{\lambda}}{d t} & =12\left[\bar{\lambda}^{2}+\left(\bar{g}_{t}^{2}-A\right) \bar{\lambda}+B-\bar{g}_{t}^{4}\right], \quad t=\ln \mu
\end{aligned}
$$

\footnotetext{
${ }^{2}$ In the symmetric phase of extremal weak coupling $G<G_{c}$, all four-fermion interacting amplitudes are suppressed by the cutoff $\Lambda$.
} 


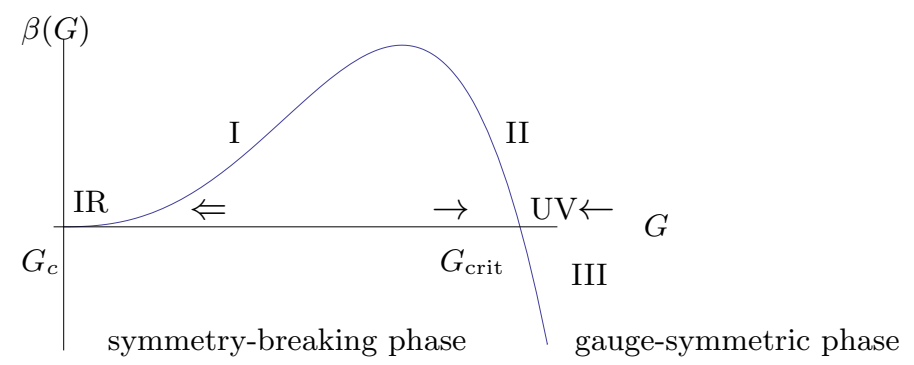

Figure 1. This is a sketch [20] to qualitatively show the behavior of the $\beta$-function in terms of the four-fermion coupling $G$. We indicate the IR-stable fixed point $G_{c}$ and a possible UV-stable fixed point $G_{\text {crit }}$, the latter separates the symmetry-breaking phase (positive $\beta(G)$-function) from the gauge-symmetric phase (negative $\beta(G)$-function). We also indicate the different parts of $\beta(G)$ function: the positively increasing part "I" and the IR-domain where RG flow approaches (thick left arrow) to the IR-fixed point, as energy scale $\mu$ decreases; (ii) the positively decreasing part "II" and the negative part "III", as well as the UV-domain where RG flow approaches (thin right and left arrows) to the UV-fixed point, as the running energy scale $\mu$ increases.

where one can find $A, B$ and RG equations for running gauge couplings $g_{1,2,3}^{2}$ in eqs. (4.7), (4.8) of ref. [28].

This IR-domain of IR-stable fixed point $G_{c}$ can be illustrated by a positive $\beta(G)$ function for $G \gtrsim G_{c}$. Taking into account one fermion-loop contribution (tadpole diagram) from the SSB-relevant top-quark channel (2.15), we calculate the gap equation for topquark mass $m_{t} \neq 0$, and obtain the $\beta$-function

$$
\beta(G) \equiv \mu \frac{d G}{d \mu} \approx 2 \frac{G^{2}}{G_{c}}\left(\frac{\mu}{\mathcal{E}}\right)^{2}\left[1+\ln \left(\frac{\mathcal{E}}{\mu}\right)^{2}\right]>0,
$$

for $G \gtrsim G_{c} \equiv 8 \pi^{2} /\left(N_{c} \mathcal{E}^{2}\right)$ and intermediate energy scale $\mathcal{E}>\mu \gtrsim v$, where $N_{c}=3$ is the number of colors. The positive $\beta$-function of eq. (3.4) indicates that an IR-stable fixed point $G_{c}$ and the IR-domain $G \rightarrow G_{c}+0^{+}$as the running energy scale $\mu \rightarrow v$, as shown the part "I" of the $\beta(G)$-function in figure 1 . As the energy scale $\mu$ decreases, indicated by a thick arrow in figure 1, the RG flow is attracted to the IR-stable fixed point, and the effective SM of particle physics in low energies is realized in this IR-domain (vicinity) of IR-stable fixed point.

\subsubsection{Resolution to the $t \bar{t}$-condensate model}

Both experimental $m_{t}$ and $m_{H}$ values of top-quark and Higgs-boson masses were unknown in the early 1990s. In order to find low-energy values $m_{t}$ and $m_{H}$ close to the IR-stable fixed point, BHL [28] imposed the compositeness conditions $\tilde{Z}_{H}(\mu)=1 / \bar{g}_{t}^{2}(\mu) \rightarrow 0$ and $\tilde{\lambda}=\bar{\lambda}(\mu) / \bar{g}_{t}^{4}(\mu) \rightarrow 0$, as energy scale $\mu \rightarrow \Lambda$ for different values of the high-energy cutoff $\Lambda$ as the boundary conditions to solve the RG equations (3.2) and (3.3). As a result, masses $m_{t}, m_{H}$ and Higgs-decay width obtained do not agree with experimental values, thus the BHL $t \bar{t}$-condensate model was firmly excluded. From the theoretical point of view, the compositeness conditions implies that the BHL composite Higgs boson is a loosely bound 

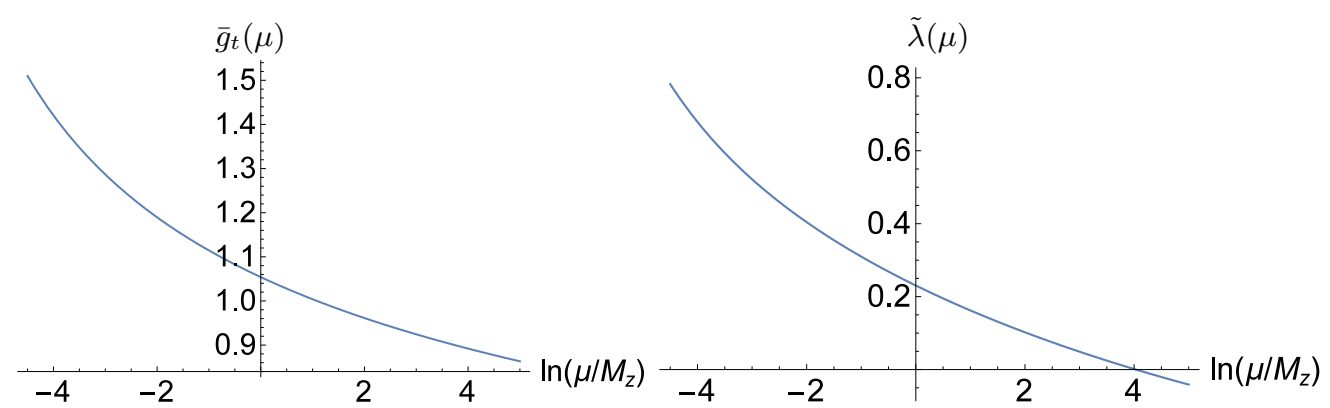

Figure 2. Using experimentally measured SM quantities (including $m_{t}$ and $m_{H}$ ) as boundary values, we uniquely solve the RG equations for the composite Higgs-boson model [28], we find [20, 51] the effective top-quark Yukawa coupling $\bar{g}_{t}(\mu)$ (left) and effective Higgs quartic coupling $\tilde{\lambda}(\mu)$ (right) in the range $1.0 \mathrm{GeV} \lesssim \mu \lesssim 13.5 \mathrm{TeV}$. Note that $\tilde{\lambda}(\mathcal{E})=0$ at $\mathcal{E} \approx 5.14 \mathrm{TeV}$ and $\tilde{\lambda}(\mu)<0$ for $\mu>\mathcal{E}$.

state of top and anti-top-quark pair $(\bar{t} t)$ because its wave-function renormalization $\tilde{Z}_{H}(\mu)$ goes to zero as $\mu \rightarrow \Lambda$.

Instead, we adopted $[20,51]$ the experimental values of the top-quark and Higgs-boson masses,

$$
m_{H}=126 \pm 0.5 \mathrm{GeV} ; \quad m_{t}=172.9 \pm 0.8 \mathrm{GeV},
$$

and used the mass-shell conditions $m_{t}=\bar{g}_{t}\left(m_{t}\right) v / \sqrt{2}$ and $m_{H}^{2} / 2=\tilde{\lambda}\left(m_{H}\right) v^{2}$, as infrared boundary conditions to integrate the RG equations (3.2) and (3.3) so as to uniquely determine the functions of top-quark Yukawa $\bar{g}_{t}(\mu)$ and Higgs-quartic $\tilde{\lambda}(\mu)$ couplings (see figure 2 ), as well as to obtain the finite values $\tilde{Z}_{H}(\mathcal{E}) \approx 1.26$ and the intermediate energy scale $\mathcal{E} \approx 5.1 \mathrm{TeV}$ for $\tilde{\lambda}(\mathcal{E})=0$. Our results (figure 2 ) are radically different from the BHL results, ${ }^{3}$ not only the completely different behaviors and numerical values of the Yukawa $\bar{g}_{t}(\mu)$ and quartic $\tilde{\lambda}(\mu)$ couplings that will be discussed in next subsection 3.1.2, but also (i) the composite Higgs boson is a tightly bound state of $\bar{t} t$ pair for finite values $\tilde{Z}_{H}(\mu)$, as if it is an elementary Higgs boson; (ii) the phase transition from the symmetry-breaking phase to the symmetric phase is indicated by $\tilde{\lambda}(\mu) \rightarrow 0^{+}$as $\mu \rightarrow \mathcal{E}+0^{-}$, will be discussed in section 3.3.1; (iii) the drastically fine-tuning (hierarchy) problem can be resolved, since the intermediate scale $\mathcal{E}(v<\mathcal{E} \ll \Lambda)$ replaces the high-energy cutoff $\Lambda$ and sets into eq. (3.4), the gap-equation

$$
\frac{1}{G_{c}}-\frac{1}{G}=\frac{1}{G_{c}}\left(\frac{m_{t}}{\mathcal{E}}\right)^{2} \ln \left(\frac{\mathcal{E}}{m_{t}}\right)^{2}>0
$$

giving rise to a soft chiral-symmetry breaking scale $m_{t} \lesssim v$ and the pseudoscalar decay constant,

$$
f_{\pi}^{2} \approx \frac{N_{c}}{32 \pi^{2}} m_{t}^{2} \ln \frac{\mathcal{E}^{2}}{m_{t}^{2}}=\frac{N_{c}}{32 \pi^{2}} \mathcal{E}^{2}\left(1-\frac{G_{c}}{G}\right)
$$

for more detailed discussions, see ref. [51].

In the IR-domain $\left(G \gtrsim G_{c}\right)$ of unique four-fermion coupling $G$, it seems that the fourfermion operators (2.15) undergo the SSB, leading to the fermion-condensation $M_{f f^{\prime}}=$

\footnotetext{
${ }^{3}$ Using the compositeness conditions $\tilde{Z}_{H}(\mu)=1 / \bar{g}_{t}^{2}(\mu) \rightarrow 0, \mu \rightarrow \Lambda$, we reproduced the BHL results: figures 4 and 5; tables I and II of ref. [28].
} 
$-G\left\langle\bar{\psi}^{f} \psi^{f^{\prime}}\right\rangle=m \delta_{f f^{\prime}} \neq 0$, two diagonal mass matrices of quark sectors $q=2 / 3$ and $q=-1 / 3$ satisfying $3+3$ mass-gap equations, see eqs. (2.11) and (2.12). It was demonstrated [50] that as an energetically favorable solution of the SSB ground state of the SM, only top-quark is massive $\left(m_{t}^{\mathrm{sb}}=-G\left\langle\bar{\psi}_{t} \psi_{t}\right\rangle \neq 0\right)$, otherwise in addition to those become the longitudinal modes of massive gauge bosons, there would be more Goldstone modes contributing the SSB-ground-state energy. In other words, among four-fermion operators (2.15) and (2.16), the $\langle\bar{t} t\rangle$-condensate model (3.1) is the unique channel undergoing the SSB of SM gauge symmetries, for the reason that this is energetically favorable, i.e., the ground-state energy is minimal when the maximal number of Goldstone modes are three and equal to the number of the longitudinal modes of massive gauge bosons in the SM. Moreover, the four-fermion operators (2.16) of the lepton sector do not undergo the SSB leading to the lepton-condensation $M_{f f^{\prime}}=-G\left\langle\bar{\ell}^{f} \ell^{f^{\prime}}\right\rangle=m_{\ell} \delta_{f f^{\prime}} \neq 0$, i.e., two diagonal mass matrices of the lepton sector $(q=0$ and $q=-1)$. The reason is that the effective four-lepton coupling $\left(G N_{c}\right) / N_{c}$ is $N_{c}$-times smaller than the four-quark coupling $\left(G N_{c}\right)$, where the color number $N_{c}=3$. In the IR-domain $\left(G \gtrsim G_{c}\right)$ of the IR-stable fixed point $G_{c}$, the effective four-quark coupling is above the critical value and the SSB occurs, whereas the effective four-lepton coupling is below the critical value and the SSB does not occur for the lepton sector.

As a result, only the top quark acquires its mass via the SSB and four-fermion operator (3.1) of the top-quark channel becomes the relevant operator following the RG equations in the IR domain [28]. While all other quarks and leptons do not acquire their masses via the SSB and their four-fermion operators (2.15), (2.16), (2.17), (2.18) are irrelevant dimension- 6 operators, whose tree-level amplitudes of four-fermion scatterings are highly suppressed $\mathcal{O}\left[(\mu / \Lambda)^{2}\right]$, thus their deviations from the SM are nowadays experimentally inaccessible [21].

\subsubsection{Experimental indications of tightly-bound composite Higgs boson?}

The wave-function renormalization $\tilde{Z}_{H}(\mu)=1 / \bar{g}_{t}^{2}(\mu)$ represents the form-factor of composite Higgs boson. In the BHL case, the compositeness condition $\tilde{Z}_{H}(\mu) \rightarrow 0, \mu \rightarrow \Lambda$ is adapted, this implies that the BHL loosely-bound composite Higgs boson behaves very differently from the elementary Higgs boson, and significant deviations from the SM are expected. In theoretical calculations, the BHL Yukawa-coupling $\bar{g}_{t}^{\mathrm{BHL}}(\mu)$ increases with energy $\mu$, and Higgs-quartic coupling $\tilde{\lambda}^{\mathrm{BHL}}(\mu)$ increases for $M_{Z}<\mu \lesssim 5 \mathrm{TeV}$ (see figures 4 and 5 of ref. [28]), which are completely different from our results of figure 2 . In experimental fact, the BHL results are firmly excluded by the measured values of Higgs mass and decay rate (width). Instead, in our case the non-vanishing $\tilde{Z}_{H}(\mu)$ means that after conventional wave-function and vertex renormalizations, the tightly-bound composite Higgs boson behaves as if an elementary particle. The deviations from the SM with an elementary Higgs boson are expected to be very small.

However, the effective top-Yukawa $\bar{g}_{t}(\mu)$ and Higgs-quartic $\tilde{\lambda}(\mu)$ couplings monotonically and slightly decrease as the energy scale $\mu$ increases over the range $\left(M_{Z}<\mu<\mathcal{E}\right)$, see figure 2. In the range $m_{H}<\mu<\mathcal{E}$, these might imply some effects on the rates or cross-sections of the following three dominate processes of Higgs-boson production and 
decay $[8,9]$ or other relevant processes. Two-gluon fusion produces a Higgs boson via a top-quark loop, which is proportional to the effective Yukawa coupling $\bar{g}_{t}(\mu)$. Then, the produced Higgs boson decays into the two-photon state by coupling to a top-quark loop, and into the four-lepton state by coupling to two massive $W$-bosons or two massive $Z$ bosons. Due to the $\bar{t} t$-composite nature of Higgs boson, the one-particle-irreducible (1PI) vertexes of Higgs-boson coupling to a top-quark loop, to two massive $W$-bosons and to two massive $Z$-bosons are proportional to the effective Yukawa coupling $\bar{g}_{t}(\mu)$. As a result, both the Higgs-boson decaying rate $\Gamma_{H \rightarrow f}$ to each of these three channels and total decay rate $\Gamma_{H}^{\text {total }}=\sum_{f} \Gamma_{H \rightarrow f}$ are proportional to $\bar{g}_{t}^{2}(\mu)$, however the branching ratio $\left(\Gamma_{H \rightarrow f} / \Gamma_{H}^{\text {total }}\right)$ of each Higgs-decay channel is not changed. The energy scale $\mu$ is actually the Higgs-boson energy, representing the total energy of final states, e.g., two-photon state and four-lepton states, into which the produced Higgs boson decays.

These discussions imply that the resonant amplitude (number of events) of two-photon invariant mass $m_{\gamma \gamma} \approx 126 \mathrm{GeV}$ and/or four-lepton invariant mass $m_{4 l} \approx 126 \mathrm{GeV}$ is expected to become smaller as the produced Higgs-boson energy $\mu$ increases, i.e., the energy of final two-photon and/or four-lepton states increases, when the CM energy $\sqrt{s}$ of LHC $p p$ collisions increases with a given luminosity. Suppose that the total decay rate or each channel decay rate of the SM Higgs boson is measured at the Higgs-boson energy $\mu=m_{t}$ and the SM value of Yukawa coupling $\bar{g}_{t}^{2}\left(m_{t}\right)=2 m_{t}^{2} / v \approx 1.04$, see figure 2 . In the scenario of tightly-bound composite Higgs boson, as the Higgs-boson energy $\mu$ increases to $\mu=2 m_{t}$, the Yukawa coupling $\bar{g}_{t}^{2}\left(2 m_{t}\right) \approx 0.98$, the variation of total decay rate or each channel decay rate is expected to be $6 \%$ for $\Delta \bar{g}_{t}^{2} \approx 0.06$. Analogously, the variation is expected to be $9 \%$ at $\mu=3 m_{t}, \bar{g}_{t}^{2}\left(3 m_{t}\right) \approx 0.95$ or $11 \%$ at $\mu=4 m_{t}, \bar{g}_{t}^{2}\left(4 m_{t}\right) \approx 0.93$, as shown in figure 2 . These variations are still too small to be clearly distinguished by the present LHC experiments. Such scenario of tightly-bound composite Higgs boson has not been found so far any tension with electroweak precision variables. Nevertheless, it certainly needs more detailed analysis of the tightly-bound composite Higgs phenomenology in comparison with electroweak precision variables in future work.

In addition, the nonresonant new phenomena, stemming from four-fermion scattering amplitudes of diemnsion-6 irrelevant operators in effective Lagrangian (2.6), are hightly suppressed $\mathcal{O}\left(\Lambda^{-2}\right)$ and thus hard to be identified [21] in high-energy processes of LHC $p p$ collisions (e.g., the Drell-Yan dilepton process, see ref. [52]), $e^{-} e^{+}$annihilation to hadrons and deep inelastic lepton-hadron $e^{-} p$ scatterings at TeV scales. Nonetheless, these effects are the nonresonant new signatures of low-energy collider that show the deviations of such scenario from the SM.

\subsubsection{Yukawa couplings and FCNC's in IR-domain}

The SSB generated top-quark mass $m_{t} \propto \bar{g}_{t} v$ breaks chiral symmetries, fermion-family mixing matrices (2.14) become relevant for coupling different fermion families. As a consequence, other quarks and leptons acquire their masses $\bar{g}_{f} v$, because Schwinger-Dyson (SD) equations for their self mass-energy functions acquire the explicit symmetry breaking (ESB) terms induced by the $W^{ \pm}$-boson vector-like couplings and quark-lepton interactions at high energies, via the fermion-family mixing matrices like the CKM (2.14) and PMNS 
matrices in the SM. References $[12,13]$ show in details how the top quark acquires its mass together with Higgs boson mass by the SSB in terms of $\bar{g}_{t}(\mu)$ and $\tilde{\lambda}(\mu)$ couplings (figures 2), and other SM fermions acquire their masses $m_{f} \propto \bar{g}_{f} v$ by the ESB, as functions of the top-quark mass $m_{t}$ or Yukawa coupling $\bar{g}_{t}(\mu)$, and mixing-matrix elements of the CKM and PMNS types in the SM, which are intrinsic parameters fixed by experiments. The hierarchy patterns of fermion masses and mixing-matrix elements are closely related.

As a consequence, the SM chiral gauge symmetries and global fermion-family (flavor) symmetries are broken at a soft energy scale $m^{f}=\bar{g}_{t} v \ll \mathcal{E} \ll \Lambda$. The latter fermion-family $U_{L}(3) \times U_{R}(3)$ symmetries are broken to the $\mathrm{U}(1)$ symmetry for each fermion family, i.e., $U_{1}(1) \times U_{2}(1) \times U_{3}(1)$. Thus the quadrilinear four-fermion operators (2.10), bilinear twofermion operators (2.11) and (2.12) are "diagonal", namely they are for each fermion family without any fermion-family mixing in the fermion-family space based on mass eigenstates of fermions. Given a definite electric charge, the U(1)-symmetry for each fermion flavor is still preserved and the quantum number of each fermion flavor is conserved. This completely prohibits any four-fermion-interacting process that violates fermion-flavor-number conservation, such as the FCNC process converting from one elementary fermion flavor $\psi^{f}$ to another $\psi^{f^{\prime}}$ with the same electric charge.

However, such prohibition is relaxed as perturbatively turning on the interactions (2.6) between chiral-gauge-boson $W^{ \pm}$and massive fermions in addition to the four-fermion interactions (2.10). The reason is that these chiral-gauge interactions are not diagonal in the fermion-family space based on the mass eigenstates of fermions (2.11) and (2.12), attributed to the fact that the mass eigenstates of the SM elementary fermions are different from their chiral-gauge eigenstates. As a result, a 1PI-interacting vertex that violates the fermion-flavor-symmetry $U_{1}(1) \times U_{2}(1) \times U_{3}(1)$ is induced via the mixing matrices of CKM type (2.14) and interactions among SM gauge bosons, e.g. $W^{ \pm}$and photon, at one-loop level. Such 1PI vertex allows the FCNC processes of changing one elementary fermion $\psi^{f}$ flavor [mass eigenstate (2.8) or (2.9)] to another elementary fermion $\psi^{f^{\prime}}$ flavor [mass eigenstate] with the same electric charge. This situation is the same as that of the SM with an elementary Higgs boson.

Nevertheless, such $W^{ \pm}$-boson contributions of SM-type to the FCNC processes are highly suppressed, since they come from the loop-level contributions of the SM $W^{ \pm}$-boson via the fermion-family mixing matrix of the CKM type (2.14) in its gauge coupling vertexes. In conclusion, apart from the SM-type suppressed contributions to FCNC, the effective Lagrangian (2.6) with four-fermion operators (2.10) in the IR-domain does not contain any additional unsuppressed 1PI vertexes that contribute to the FCNC processes.

\subsection{Gauge-symmetric phase of strong four-fermion coupling}

We turn to the brief recall on the gauge-symmetric phase of strong four-fermion coupling $G$,

$$
-G \bar{\psi}_{L} \psi_{R} \bar{\psi}_{R} \psi_{L}
$$

preserving $S U_{L}(2)$ gauge symmetry, where two-component Weyl fermion $\psi_{L}\left(\psi_{R}\right)$ is an $S U_{L}(2)$ doublet (singlet). The strong-coupling expansion was adopted to calculate twopoint functions of composite boson and fermion fields [14, 15], and the vertex functions of 
their couplings to the $S U_{L}(2)$-gauge bosons [16-19]. Detailed calculations can be found in these references and their appendices. In the lowest non-trivial order, we obtained the propagators of the massive composite bosons $\mathcal{A}=\left(\bar{\psi}_{R} \psi_{L}\right)$ and composite Dirac fermions: $\Psi_{D}=\left(\psi_{L}, \mathcal{A} \psi_{R}\right)$ and $\Psi_{S}=\left(\mathcal{A}^{\dagger} \psi_{L}, \psi_{R}\right)$,

$$
\left\langle\mathcal{A}(0) \mathcal{A}^{\dagger}(x)\right\rangle \Rightarrow \frac{Z_{B}^{S}}{q^{2}+M_{B}^{2}} ; \quad\left\langle\Psi_{D, S}(0) \bar{\Psi}_{D, S}(x)\right\rangle \Rightarrow Z_{D, S}^{S} \frac{i p^{\mu} \gamma_{\mu}+M_{D, S}}{p^{2}+M_{D, S}^{2}},
$$

where the pole $M_{B, D, S}$ and residue $Z_{B, D, S}^{S}$ respectively represent mass and form factor of composite particles. As long as their form-factors are finite, these composite particles behave as elementary particles. The propagators of renormalized composite particles (3.9) give their mass-shell conditions,

$$
E_{B}=\sqrt{q^{2}+M_{B}^{2}} \approx M_{B}, \quad E_{D, S}=\sqrt{p^{2}+M_{D, S}^{2}} \approx M_{D, S}, \quad \text { for } q^{2}, p^{2} \ll M_{B, D, S}^{2} .
$$

The vertex functions of their $S U_{L}(2)$-couplings were obtained by Ward identities from propagators (3.9), because the composite-particle spectra preserve the $S U_{L}(2)$ gauge symmetry.

These massive composite particles formed by the such strong-coupling dynamics in the gauge-symmetric phase are completely different from the composite particles like massive Higgs boson and massless Goldstone bosons formed by the weak-coupling NJL dynamics in the symmetry-breaking phase where the SSB takes place.

\subsection{Strong critical coupling and the UV-stable fixed point}

This indicates that there are two distinct phases: (i) the symmetry-breaking phase $G_{c}<$ $G<G_{\text {crit }}$ for the SM of elementray particles; (ii) the gauge-symmetric phase $G>G_{\text {crit }}$ for an effective theory of composite particles, and the second-order phase transition from one phase to another at the strong critical coupling $G_{\text {crit }}$.

\subsubsection{Strong critical coupling}

In the symmetric phase, we indeed found $[14,15]$ the existence of strong critical coupling $G_{\text {crit }}$ by using strong four-fermion coupling expansion to approximately calculate the twopoint Green function (3.9) of composite boson and examine sign change of quadratic term $M_{B}^{2} \mathcal{A} \mathcal{A}^{\dagger}(x)$. Instead, based on the symmetry-breaking phase of weak four-fermion coupling, see section 3.1, we show the existence of strong critical point $G_{\text {crit }}$ of the second-order phase transition from the symmetry-breaking phase to gauge-symmetric phases.

In refs. [20, 51], we solve the full one-loop RG equations [28] for running couplings $\bar{g}_{t}\left(\mu^{2}\right)$ and $\bar{\lambda}\left(\mu^{2}\right)$ with the top-quark and Higgs-boson mass-shell conditions

$$
m_{t}=\bar{g}_{t}\left(m_{t}\right) v / \sqrt{2}, \quad m_{H}^{2} / 2=\tilde{\lambda}\left(m_{H}\right) v^{2}
$$

$m_{t} \approx 172.9 \mathrm{GeV}$ and $m_{H} \approx 126 \mathrm{GeV}$ fixed by experiments. As a result, we uniquely determine the renormalized form factor $\tilde{Z}_{H}(\mu)$ and quartic coupling $\tilde{\lambda}(\mu)$ of the composite 
Higgs particle. We also find the intermediate energy scale $\mathcal{E}$, at which the effective quartic coupling $\tilde{\lambda}(\mathcal{E})=0$, and the finite value of form-factor $\tilde{Z}_{H}(\mathcal{E})$,

$$
\mathcal{E} \approx 5.1 \mathrm{TeV}, \quad \tilde{Z}_{H}(\mathcal{E}) \approx 1.26, \quad \tilde{\lambda}(\mathcal{E})=0
$$

otherwise the effective theory would run into an instability $\left(\tilde{\lambda} \sim 0^{-}\right)$beyond $\mathcal{E}$. These are certainly preliminary and qualitative results, since we use one-loop RG equations in lowenergy and weak-coupling region and extrapolate their solutions to high-energy and strongcoupling region. Nevertheless, the solution (3.12) implies the following three important features. (i) In the effective Lagrangian of quadratic term $m_{H}^{2} H H^{\dagger} / 2$, the squared Higgsboson mass $m_{H}^{2}=2 \tilde{\lambda}(\mu) v^{2}$ changes its sign at $\mu=\mathcal{E}$, indicating the second-order phase transition $G_{\text {crit }}$ from the symmetry-breaking phase to a gauge-symmetric phase of highenergy and strong-coupling region. (ii) The form-factor $\tilde{Z}_{H}(\mu) \neq 0$ shows that the tightly bound composite Higgs particle behaves as if an elementary particle for $\mu \leq \mathcal{E}$. (iii) The effective form-factor $\tilde{Z}_{H}(\mathcal{E})$ of composite Higgs boson is finite, implying the formation of massive composite fermions $\Psi \sim(H \psi)$ in the gauge-symmetric phase.

To close this subsection, it is worthwhile to mention that in ref. [53] it is shown in the elementary Higgs-boson model that the quadratic term from high-order quantum corrections has a physical impact on the SSB and the phase transition to a symmetric phase occurs at the scale of order of $\mathrm{TeV}$.

\subsubsection{UV-stable fixed point}

In order to show that this critical point $G_{\text {crit }}$ of the second-order phase transition can be a UV-fixed point, we calculated the $\beta$-function in the symmetric phase of strong fourfermion coupling. Up to the lowest non-trivial order of the strong coupling expansion, i.e., the two-fermion-loop contribution (sun-set diagrams), and we obtained the negative $\beta$-function [20]

$$
\beta(G)=p^{2} \frac{\partial \mathcal{G}(p)}{\partial p^{2}} \approx \frac{6}{\mathcal{G}} \frac{\partial \Phi\left(p^{2} / \Lambda^{2}\right)}{\partial \ln \left(p^{2} / \Lambda^{2}\right)}<0,
$$

where $\mathcal{G} \equiv G \times(\Lambda / \pi)^{2}$ and the dimensionless Lorentz-scalar function $\Phi\left(p^{2} / \Lambda^{2}\right)$ is positive and finite, monotonically decreases as the energy scale $p^{2} / \Lambda^{2}$ increases. On the basis of the $\beta(G)$-function being positive (3.4) and becoming negative (3.13), as sketched as "I", "II" and "III" in figure 1, we infer there must be at least one zero-point of the $\beta(G)$ function, i.e., $\beta\left(G_{\text {zero }}\right)=0$ and $\beta^{\prime}\left(G_{\text {zero }}\right)<0$. This zero-point $G_{\text {zero }}$ is a UV-stable fixed point, which should coincide with the strong critical coupling $G_{\text {zero }} \approx G_{\text {crit }}$ for the reason that a UV-stable fixed point should be the candidate of the critical point $G_{\text {crit }}$ for the second-order phase transition from the symmetry-breaking phase to the symmetric phase. As the running energy scale $\mu$ increases, the effective coupling $G(\mu) \rightarrow G_{\text {crit }} \pm 0$ (thin right and left arrows, see figure 1) is attracted to this UV-stable fixed point from both symmetry-breaking and symmetric phases. In the scaling region (vicinity) of UV-stable fixed point $G_{\text {crit }}$, i.e., UV-domain, RG flow approaches to the UV-fixed point, as energy scale $\mu$ increases, in the scenes of renormalization group (scaling) invariance and resultant RG equations. 
This is analogous to non-linear $\sigma$ models [54], which contain a dimensionful coupling constant and are thus not perturbatively renormalizable. Nevertheless, the nonperturbative critical coupling of these $\sigma$ models shows the second-order phase transition from the ordered (symmetry-breaking) phase to the disordered (symmetric) phase, and exhibits a non-trivial UV-fixed point and UV-scaling domain of the renormalization group both in the lattice formulation $[55,56]$ and in the $2+\epsilon$ formulation [57, 58].

However, due to the lack of a non-perturbative method to effectively approach to the critical coupling $G_{\text {crit }}$ and its neighborhood, we have not been able to quantitatively determine the $G_{\text {crit }}$ value and properties of its scaling region i.e., the UV-domain, which will be qualitatively discussed and recalled in next section.

\subsection{The UV-domain of UV-stable fixed point}

\subsubsection{Energy threshold of composite particles}

As the running energy scale $\mu$ decreases in the vicinity of UV-stable fixed point $G_{\text {crit }}$, the $\beta(\mu)$ function (see figure 1) shows that the RG flows take the effective theory of composite particles away from the UV fixed point towards the IR-domain of the IR-fixed point $G_{c}$, where the low-energy SM of elementary particle physics is realized. This implies the existence of the energy threshold $\mathcal{E}_{\text {thre }}$, below which $\mu<\mathcal{E}_{\text {thre }}$ composite particle dissolves into its constituents of SM elementary particles. As discussed in sections $\mathrm{V}$ and VI of refs. $[17,18]$, when the energy scale $\mu$ decreases to the energy threshold $\mathcal{E}_{\text {thre }}$ and $G(\mu) \rightarrow G_{\text {crit }}\left(\mathcal{E}_{\text {thre }}\right)$, the phase transition occurs from the symmetric phase to the symmetry-breaking phase, all composite particles (poles) dissolve into their constituents, which are represented by three-fermion, fermion-boson and two-fermion cuts in the energymomentum plane, as their form factor and binding energy vanish [59-62]. Actually, the energy threshold $\mathcal{E}_{\text {thre }}$ represents the symmetry-breaking scale at the second-order phase transition $G_{\text {crit }}$.

The composite-particle masses $M(3.10)$ contain the negative binding energy $-\mathcal{B}[G(\mu)]$ and positive kinetic energies $\mathcal{K}$ of their constituents. The energy threshold $\mathcal{E}_{\text {thre }}$ is determined by $\mathcal{B}[G(\mu)]_{\mu \rightarrow \mathcal{E}_{\text {thre }}} \rightarrow \mathcal{K}$ and vanishing form factors of composite particles in the symmetric phase. These calculations require non-perturbative approaches that need to be developed in future. Nevertheless, we gain some physical insight into the symmetrybreaking scale $\mathcal{E}_{\text {thre }} \gtrsim \mathcal{E} \approx 5.1 \mathrm{GeV}$, since the approximate $\mathcal{E}$-value (3.12) is obtained in symmetry-breaking phase by extrapolating the solutions of the one-loop RG-equations of low-energy $(v)$ region to high-energy $(\mathcal{E})$ region.

\subsubsection{Characterisic energy scale}

In the IR-domain of the fixed point $G_{c}$ where the effective Lagrangian of the SM is realized, the correlation length $\xi_{v}$ corresponding to the electroweak scale $v=239.5 \mathrm{GeV}$ that is attributed to the SSB dynamics in symmetry-breaking phase sets the physical energy scale of IR-domain. This characteristic energy scale is much smaller than the cutoff $(v \ll \Lambda)$, leading to the renormalization group (scaling) invariance and resultant RG equations in the IR-domain. This characteristic electroweak scale $v$ is determined by the experimental measurements of intermediate gauge bosons $W^{ \pm}$and $Z^{0}$ masses. 
Analogously, as discussed in ref. [20], in the UV-domain (scaling region) of the UVfixed point (critical point) $G_{\text {crit }}$ of the second-order phase transition, the correlation length $\xi$ or characteristic energy $\mathcal{E}_{\xi}=\xi^{-1}$ sets the physical scale. The correlation length $\xi$ is much larger than the cutoff $a \approx \Lambda^{-1}$, i.e., $\xi \gg a$ or $\mathcal{E}_{\xi} \ll \Lambda$ leads to the renormalization group (scaling) invariance and resultant RG equations in the UV-domain. In this scaling domain, the running coupling $G(a / \xi)$ can be expanded as a series,

$$
G(a / \xi)=G_{\text {crit }}\left[1+a_{0}(a / \xi)^{1 / \nu}+\mathcal{O}\left[(a / \xi)^{2 / \nu}\right]\right] \rightarrow G_{\text {crit }}+0^{+},
$$

for $a / \xi \ll 1$, leading to the $\beta$-function

$$
\beta(G)=(-1 / \nu)\left(G-G_{\text {crit }}\right)+\mathcal{O}\left[\left(G-G_{\text {crit }}\right)^{2}\right]<0 .
$$

The correlation length $\xi$ follows the scaling law

$$
\xi=c_{0} a \exp \int^{G} \frac{d G^{\prime}}{\beta\left(G^{\prime}\right)}=\frac{c_{0} a}{\left(G-G_{\text {crit }}\right)^{\nu}},
$$

where the coefficient $c_{0}=\left(a_{0} G_{\text {crit }}\right)^{\nu}$ and critical exponent $\nu$ need to be determined by non-perturbative numerical simulations. The physical scale $\mathcal{E}_{\xi} \equiv \xi^{-1}$ in the UV-domain is attributed to the strong-coupling dynamics of forming composite particles. This implies the masses of composite particles (3.9) and (3.10)

$$
M \propto \mathcal{E}_{\xi}=\xi^{-1},
$$

and the running coupling $\left.G(\mu)\right|_{\mu \rightarrow \mathcal{E}_{\text {thre }}+0^{+}} \rightarrow G_{\text {crit }}$,

$$
G(\mu) \simeq G_{\text {crit }}\left[1-\frac{1}{\nu} \ln \left(\frac{\mu}{\mathcal{E}_{\xi}}\right)\right]^{-1}, \quad \mu / \mathcal{E}_{\xi}=\xi /\left(a a_{0}^{\nu}\right)>1,
$$

and the scale $\mu$ indicates the energy transfer between constituents inside composite particles.

On the basis of these discussions and observations, we advocate the following relation for (i) the energy scale $\mathcal{E} \approx 5 \mathrm{TeV}$ of eq. (3.12) extrapolated by the RG equations in the IR-domain for the SM, (ii) the energy threshold $\mathcal{E}_{\text {thre }}$ corresponding to the phase-transition scale from symmetric to symmetry-breaking phases and (iii) the characteristic energy scale $\mathcal{E}_{\xi}$ in the UV-domain for the effective theory of composite particles

$$
\mathcal{E} \lesssim \mathcal{E}_{\text {thre }} \lesssim \mathcal{E}_{\xi} \ll \Lambda, \quad \mathcal{E} \approx 5 \mathrm{TeV}
$$

The values of these characteristic scales of UV-domain need some experimental knowledge in high energies, analogously to the electroweak scale $(v)$ of IR-domain.

\subsection{Relevant and irrelevant operators in IR- and UV-domains}

In the weak-coupling IR-domain, as an energetically favorable solution for the SSB ground state [50], among four-fermion operators (2.6) the top-quark channel $G\left(\bar{\psi}_{L}^{i a} t_{R a}\right)\left(\bar{t}_{R}^{b} \psi_{L i b}\right)[28]$ is the only physically relevant and renormalizable operators of effective dimension- 4 , due 
to the NJL-dynamics for the SSB. Namely, it becomes the effective SM Lagrangian (3.1) with bilinear top-quark mass term and Yukawa coupling to the composite Higgs boson $H$, which obeys the RG equation approaching to the low-energy SM physics characterized by the energy scale $v \approx 239.5 \mathrm{GeV}$. Other four-fermion operators in eq. (2.6), as well as repulsive four-fermion operators (2.4), do not undergo the SSB and are irrelevant dimension-6 operators, whose tree-level amplitudes of four-fermion scatterings are suppressed $\mathcal{O}\left(\Lambda^{-2}\right)$, thus their deviations from the SM could not be experimentally accessible today.

In the strong-coupling UV-domain, all attractive four-fermion operators (2.6) are physically relevant operators associating to the strong-coupling dynamics and formation of composite bosons and fermions (3.9). Due to the unique four-fermion coupling $G$, this strong-coupling dynamics of forming composite particles occurs in all channels of eq. (2.6) for each SM fermion family. These composite particles on mass-shells behave as if they were elementary, as long as their form factors are finite. All 1PI functions $\Gamma[\mu, G(\mu)]$ of the quantum field theory (2.6) at the UV-cutoff scale $\Lambda$ evolve to either irrelevant or relevant 1PI functions, as the running energy scale $\mu$ increases. The irrelevant 1PI functions are suppressed by powers of $\left(\mathcal{E}_{\xi} / \Lambda\right)^{n}$ and thus decouple from the effective theory. Instead, the relevant 1PI functions follow the scaling law (RG equations), therefore are effectively dimension- 4 and renormalizable, for example the propagators of composite fermions and bosons and their vector-like coupling vertexes to the SM gauge bosons. The effective field theory in this UV-domain is expected to contains the massive composite particle spectrum preserving the SM chiral gauge symmetries, and relevant operators of effective dimension- 4 following the RG equations. Compared with the SM in the IR-domain, the effective theory of composite particles in the UV-domain has the same chiral gauge symmetries (quantum numbers) and couplings to gauge bosons $\left(\gamma, W^{ \pm}, Z^{0}\right.$ and gluon), but the different vectorlike spectra and coupling vertexes, apart from massive composite particles being comprised by SM elementary particles. Some of these peculiar features and their possible experimental implications were studied in refs. [12, 20, 21]. These are the properties of effective composite-particle theory in the UV-domain that we attempt to study in this article and in future.

To end this section, it is worthwhile to note that the repulsive four-fermion operators (2.4) are irrelevant operators of dimension-6, and thus suppressed $\mathcal{O}\left(\Lambda^{-2}\right)$ for the reasons that they are neither associated with the NJL dynamics of the SSB in the IRdomain, nor associated with the strong-coupling dynamics of forming composite particles in the UV-domain.

It is also worthwhile to mention that these discussions are reminiscent of the asymptotic safety $[63,64]$ that quantum field theories regularized at UV-cutoff $\Lambda$ might have a nontrivial UV-stable fixed point, $\mathrm{RG}$ flows are attracted into the UV-stable fixed point with a finite number of physically renormalizable operators. The weak and strong four-fermion coupling $G$ brings us into two distinct domains. This lets us also recall the quantum chromodynamics (QCD): asymptotically free quark states in the domain of a UV-stable fixed point and bound hadron states in the domain of a possible IR-stable fixed point. ${ }^{4}$

\footnotetext{
${ }^{4}$ The references on this issue can be found in the recent paper: Z.-Y. Zheng and G.-L. Zhou, "A method for getting well-defined coupling constant in all region", arXiv:1705.07430.
} 


\section{Composite particles and effective Lagrangian in UV-domain}

In the gauge symmetric phase of an $S U_{L}(2)$-chiral gauge theory containing the four-fermion operator (3.8), the composite particles and 1PI vertex functions, as well as their properties in the UV-domain were analyzed by using the approach of strong-coupling expansion to the dynamics of four-fermion operators at the cutoff $\Lambda$ [14, 15, 17-21]. In this section, however, based on the effective Einstein-Cartan Lagrangian (2.6) in the SM framework, see section 2.4, we are going to show the composite-particle spectrum and 1PI interaction (effective Lagrangian) in terms of the quantum numbers of SM gauge symmetries. The purpose is that we shall go further to discuss composite particles decay and other processes into SM elementary gauge bosons and fermions, relevantly to possible high-energy experiments. These are the main results of this article presented in this section and following sections 5,6 and 7 .

\subsection{Composite particles in UV-domain}

\subsubsection{Quark sector}

Performing strong-coupling calculations similar to those detailedly presented in the refs. $[14,15]$ and their appendices, we obtain the following results. For the $u$-quark channel, the massive composite boson is an $S U_{L}(2)$-doublet

$$
\mathcal{A}^{i}=\left[Z_{\Pi}^{S}\right]^{-1 / 2}\left(\bar{u}_{R a} \psi_{L}^{i a}\right)
$$

of the hypercharge $Y=-1 / 2$ and $i(a)$ being the weak isospin (color) index. This composite boson combines with an elementary two-component Weyl quark to form the composite twocomponent Weyl-fermion states (three-fermion states),

$$
\mathbf{\Psi}_{R}^{i b}=\left(Z_{R}^{S}\right)^{-1} \mathcal{A}^{i} u_{R}^{b} ; \quad \mathbf{\Psi}_{L}^{b}=\left(Z_{L}^{S}\right)^{-1} \mathcal{A}^{i \dagger} \psi_{i L}^{b},
$$

and to form the massive composite four-component Dirac-fermion states: the $S U_{L}(2)$ doublet $\boldsymbol{\Psi}_{D}^{i b}$ and the $S U_{L}(2)$ singlet $\boldsymbol{\Psi}_{S}^{b},{ }^{5}$

$$
\boldsymbol{\Psi}_{D}^{i b}=\left(\psi_{L}^{i b}, \mathbf{\Psi}_{R}^{i b}\right), \quad \mathbf{\Psi}_{S}^{b}=\left(\boldsymbol{\Psi}_{L}^{b}, u_{R}^{b}\right) .
$$

The form factors $\left[Z_{\Pi}^{S}\right]^{1 / 2}(4.1)$ and $Z_{R, L}^{S}(4.2)$ are generalized wave-function renormalizations of the composite boson and fermion operators.

The four-component composite Dirac fermions are vector-like spectra, fully preserving the parity symmetry. The composite $S U_{L}(2)$ doublets $\Psi_{D}^{i b}$ carry the same $U_{Y}(1)$ hypercharge as the SM elementary and left-handed $S U_{L}(2)$ doublets $\psi_{L}^{i b}$. The composite $S U_{L}(2)$ singlets $\Psi_{S}^{b}$ carry the same $U_{Y}(1)$-hypercharge as the SM elementary and right-handed $S U_{L}(2)$ singlets $u_{R}^{b}$. These composite particles have the SM-gauge symmetric masses

$$
M_{\Pi}^{2} \mathcal{A}^{i \dagger} \mathcal{A}^{i}, \quad M_{F} \overline{\mathbf{\Psi}}_{D}^{i b} \Psi_{D}^{i b}, \quad \text { and } \quad M_{F} \overline{\mathbf{\Psi}}_{S}^{b} \boldsymbol{\Psi}_{S}^{b}
$$

\footnotetext{
${ }^{5}$ A four-component composite Dirac fermion composes an elementary Weyl fermion and a composite Weyl fermion, each of them is two-component, either left- or right-handed (a definite chirality ) [14, 15], similar discussions for massive composite bosons, see ref. [20].
} 
For the $d$-quark channel, the composite particles are represented by eqs. (4.1)-(4.3) with the replacement $u_{R a} \rightarrow d_{R a}$. In this case, $\mathcal{A}^{i}$ has the $U_{Y}(1)$-hypercharge $Y=1 / 2 . \Psi_{L}^{b}$ and $\boldsymbol{\Psi}_{S}^{b}$ have the same $U_{Y}(1)$-hypercharge as the quark field $d_{R}^{b} . \boldsymbol{\Psi}_{R}^{i b}$ and $\boldsymbol{\Psi}_{D}^{i b}$ have the same $U_{Y}(1)$-hypercharge, i.e., the $U_{Y}(1)$-hypercharge of the SM quark fields $\psi_{L}^{i b}$.

This represents the first family of the composite particles composed by the SM elementary particles (quarks) in the first family. The composite boson and fermion states (4.1)(4.3) are the eigenstates of both the SM gauge interactions and mass operators (4.4), due to the SM gauge symmetries are preserved by the massive spectra of composite particles. The same discussions also apply for the second and third quark families by substituting the $S U_{L}(2)$ doublet $\left(u_{L a}, d_{L a}\right)$ into $\left(c_{L a}, s_{L a}\right)$ or $\left(t_{L a}, b_{L a}\right)$ and singlet $u_{R a}$ into $t_{R a}$ or $c_{R a}$, as well as singlet $d_{R a}$ into $b_{R a}$ or $s_{R a}$.

\subsubsection{Lepton sector}

In the lepton sector, the composite boson and Weyl-fermion states formed by the first term $\left(\ell_{R^{-}}\right.$channel) of eq. $(2.16)$ are:

$$
\mathcal{A}^{i}=\left[Z_{H}^{S}\right]^{-1 / 2}\left(\bar{\ell}_{R} \ell_{L}^{i}\right) ; \quad \mathbf{\Psi}_{R}^{i}=\left[Z_{R}^{S}\right]^{-1} \mathcal{A}^{i} \ell_{R}, \quad \boldsymbol{\Psi}_{L}=\left[Z_{L}^{S}\right]^{-1} \mathcal{A}^{i \dagger} \ell_{i L},
$$

and the massive composite Dirac fermions $\boldsymbol{\Psi}_{D}^{i}=\left(\ell_{L}^{i}, \boldsymbol{\Psi}_{R}^{i}\right)$ and $\boldsymbol{\Psi}_{S}=\left(\boldsymbol{\Psi}_{L}, \ell_{R}\right)$. The composite boson doublets $\mathcal{A}^{i}=\left[Z_{H}^{S}\right]^{1 / 2}\left(\bar{\ell}_{R} \ell_{L}^{i}\right)$ carry the hypercharge $Y=1 / 2$, the doublets $\Psi_{D}^{i}=\left(\ell_{L}^{i}, \mathbf{\Psi}_{R}^{i}\right)$ carry the $U_{Y}(1)$ hypercharge of the SM field $\ell_{L}^{i}$ and the singlets $\boldsymbol{\Psi}_{S}=\left(\boldsymbol{\Psi}_{L}, \ell_{R}\right)$ carry the $U_{Y}(1)$ hypercharge of the SM field $\ell_{R}$. Compared with their counterparts (4.1)-(4.3) in the quark sector, these massive composite particles have different quantum numbers of the SM gauge symmetries. The composite particles formed

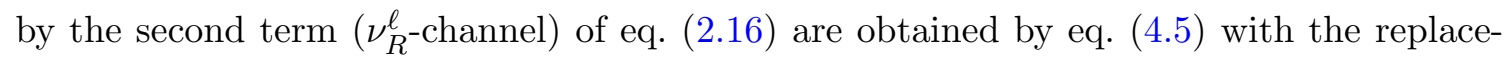
ment $\ell_{R} \rightarrow \nu_{R}^{\ell}$. In this case, $\mathcal{A}^{i}$ has the $U_{Y}(1)$-hypercharge $Y=-1 / 2 . \quad \boldsymbol{\Psi}_{L}$ and $\boldsymbol{\Psi}_{S}$ have the $U_{Y}(1)$ hypercharge of the right-handed neutrino $\nu_{R} . \Psi_{L}^{i}$ and $\Psi_{D}^{i}$ have the same $U_{Y}(1)$ hypercharge, i.e., the $U_{Y}(1)$ hypercharge of the SM lepton field $\ell_{L}^{i}$.

\subsubsection{Quark-lepton sector}

Analogously, we present for the $d_{R^{-}}^{a}$ and $e_{R^{-}}$channel of quark-lepton interactions (2.18), the massive composite Dirac fermions: $S U_{L}(2)$ doublet $\mathbf{\Psi}_{D}^{i}=\left(\ell_{L}^{i}, \mathbf{\Psi}_{R}^{i}\right)$ and singlet $\boldsymbol{\Psi}_{S}=$ $\left(\boldsymbol{\Psi}_{L}, e_{R}\right)$, where the renormalized composite boson and composite Weyl-fermion states are:

$$
\mathcal{A}^{i}=\left[Z_{H}^{S}\right]^{-1 / 2}\left(\bar{d}_{R}^{a} \psi_{L a}^{i}\right) ; \quad \mathbf{\Psi}_{R}^{i}=\left[Z_{R}^{S}\right]^{-1} \mathcal{A}^{i} e_{R}, \quad \mathbf{\Psi}_{L}=\left[Z_{L}^{S}\right]^{-1} \mathcal{A}^{i \dagger} \ell_{i L},
$$

which respectively carry the $U_{Y}(1)$-hypercharge: $Y=1 / 2, Y=-1 / 2$ and $Y=-1$. For the $\nu_{R}^{e}$-channel, the composite particles are represented by eq. (4.6) with the replacements $e_{R} \rightarrow \nu_{R}^{e}$ and $d_{R}^{a} \rightarrow u_{R}^{a}$. In this case, the counterparts of the composite states (4.6) respectively carry the $U_{Y}(1)$-hypercharge: $Y=-1 / 2, Y=-1 / 2$ and $Y=0$.

The composite particles from the second and third lepton families can be obtained by substitutions: $e \rightarrow \mu, \tau, \nu^{e} \rightarrow \nu^{\mu}, \nu^{\tau}$, and $u \rightarrow c, t$ and $d \rightarrow s, b$. In general, the

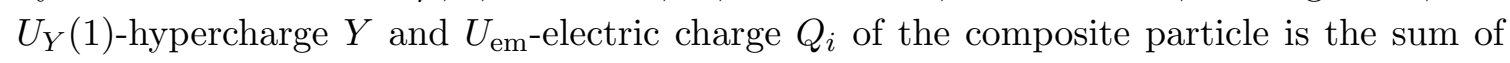
its constituents' hypercharges and electric charges, obeying the relation $Q_{i}=Y+t_{3 L}^{i}$ in units of $e$, where $t_{3 L}^{i}$ is the diagonal third component of $S U_{L}(2)$-isospin, $t_{3 L}^{1}=1 / 2$ for the neutrino and up quarks, and $t_{3 L}^{2}=-1 / 2$ for the electron and down quarks. 


\subsubsection{Discussions and three-family replication}

In the quark and lepton sectors, the massive composite boson $\mathcal{A}^{i}$, the massive composite Dirac fermions $S U_{L}(2)$ doublet $\Psi_{D}^{i}$ and singlet $\Psi_{S}$ carry an electric charge $Q=$ $(2 / 3,-1 / 3,-1,0)$ for $u_{R^{-}}^{a}$ and $d_{R^{-}}^{a}$ quark channels, $e_{R^{-}}$and $\nu_{R^{-}}$-lepton channels respectively. In the quark-lepton sector, the massive composite boson $\mathcal{A}^{i}$, the massive composite Dirac fermions $S U_{L}(2)$ doublet $\Psi_{D}^{i}$ and singlet $\Psi_{S}$ carry an electric charge $Q=(2 / 3,-1 / 3,-1,0)$ for the $d_{R^{-}}^{a} e_{R}$ channel and $u_{R}^{a}{ }^{-} \nu_{R}$ channel respectively. It should be mentioned that the composite bosons $\mathcal{A}^{i}$ carry neither the baryon number nor the lepton number, whereas the composite fermions $\Psi_{D}^{i}$ and $\Psi_{S}$ carry the baryon or the lepton number of the SM elementary fermions.

These first-family composite particles are composed only by the SM elementary particles in the first family, and there are no extra elementary gauge bosons and fermions, except the right-handed sterile neutrino $\nu_{R}$. The second-family (third-family) composite particles are composed only by the SM elementary particles in the second (third) family. The three families of composite particles are replicated corresponding to the three families of elementary fermions in the SM.

Though composite particles are massive, they carry the quantum numbers of the SM chiral gauge symmetries, which are the sum of quantum numbers of their constituents of the SM elementary particles. The propagators of these composite particles have poles and residues that respectively represent their masses and form factors [14-18]. As long as their form factors are finite, these composite particles behave as elementary particles. It should be mentioned that the gauge symmetric masses $M_{\Pi, F}$ and form factors $Z_{\Pi, L, R}^{S}$ of composite particles, e.g., (4.1)-(4.4) can be different from one to another, due to some other effects that we do not study here.

To end this section, we present some discussions on the third-family composite bosons (4.1) by the top and bottom quarks, compared with the composite Higgs and Goldstone bosons. The former comprises of the massive bound states $(\bar{t} t)^{s},\left(\bar{t} \gamma_{5} t\right)^{s}$ and $\left(\bar{t} \gamma_{5} b\right)^{s}$ formed by the strong-coupling dynamics in the UV-domain of the symmetric phase. They are different from the composite massive Higgs boson $H=(\bar{t} t)$ and massless Goldstone bosons $\left(\bar{t} \gamma_{5} t\right)$ and $\left(\bar{t} \gamma_{5} b\right)[28]$ formed by the NJL dynamics in the IR-domain of the symmetry-breaking phase. Nevertheless, at the strong critical point $G_{\text {crit }}$ of second-order phase transition that may occur around the scale $\mathcal{E}_{\xi}$, see eq. (3.12), from the point view of the different ground states of the symmetric phase and the symmetry-breaking phase, we expect some relationships of the binding energies and form factors between two types of composite bosons, e.g., $(\bar{t} t)^{s}$ and $(\bar{t} t)$, with the same SM quantum numbers. We will study this issue and its experimental implications in future.

\subsection{Effective Lagrangian of composite particles in UV-domain}

\subsubsection{SM effective Lagrangian of elementary particles in IR-domain}

To compare and contrast with the effective Lagrangian of composite particles in the UVdomain of the symmetric phase, we also present the effective SM Lagrangian in the IRdomain of the symmetry-breaking phase. The well-studied SM effective Lagrangian of 
elementary fermions in the first family reads

$$
\begin{aligned}
\mathcal{L}_{\mathrm{SM}}= & \sum_{i} \bar{\psi}^{i}\left(i \gamma_{\mu} \partial_{\mu}-m_{i}-m_{i} H / v\right) \psi^{i}-e \sum_{i} Q_{i} \bar{\psi}^{i} \gamma^{\mu} \psi^{i} A_{\mu} \\
& -\frac{g_{2}}{2 \sqrt{2}} \sum_{i} \bar{\psi}^{i} \gamma^{\mu}\left(1-\gamma_{5}\right)\left(T^{+} W_{\mu}^{+}+T^{-} W_{\mu}^{-}\right) \psi^{i} \\
& -\frac{g_{2}}{2 \cos \theta_{W}} \sum_{i} \bar{\psi}^{i} \gamma^{\mu}\left(g_{V}^{i}-g_{A}^{i} \gamma_{5}\right) \psi^{i} Z_{\mu}+\text { Higgs sector }
\end{aligned}
$$

for the both quark and lepton sector in one SM family, where $i$ is the $S U_{L}(2)$-isospin index, $T^{ \pm}$is the weak isospin raising and lowering operators $g_{V}^{i}=t_{3 L}^{i}-Q_{i} \sin ^{2} \theta_{W}$ and $g_{A}^{i}=t_{3 L}^{i}$. The weak angle $\theta_{W}=\tan ^{-1}\left(g_{1} / g_{2}\right)$ and the electron electric charge $e=g_{2} \sin \theta_{W}$. These massive fermions couple to the massless photon $A_{\mu}$, massive intermediate gauge bosons $W_{\mu}^{ \pm}$and $Z_{\mu}$. The gauge and mass eigenstates of the SM elementary fermions are different, leading to the fermion-family mixing, when three fermion families are considered. The phenomenological Yukawa couplings $\bar{g}_{i}=m_{i} / v$ of Higgs boson and elementray fermion $\psi_{i}$ of mass $m_{i}$ are related to the top-quark Yukawa coupling $\bar{g}_{t}$ of eq. (3.1) discussed in section 3.1 , and see refs. $[12,13]$ for some details.

\subsubsection{Effective Lagrangian of composite particles in UV-domain}

In the UV-domain of the symmetric phase, massive composite bosons and fermions (see section 4.1) have the quantum numbers of SM chiral gauge symmetries $S U_{c}(3) \times S U_{L}(2) \times$ $U_{Y}(1)$, couplings $g_{1}, g_{2}, g_{3}$ to SM gauge bosons $\gamma, W^{ \pm}, Z^{0}$ and gluon. The spectrum of these massive composite bosons and fermions preserves the SM chiral gauge symmetries. The gauge and mass eigenstates of massive composite bosons and fermions are the same. The interacting 1PI vertexes of composite particles and SM gauge bosons can be obtained by Ward identities associating to SM gauge symmetries. ${ }^{6}$ As a result, at tree-level we obtain the following effective Lagrangian for the first-family composite particles (4.1), (4.3), (4.5), (4.6).

For the massive composite Dirac fermions $\Psi_{D}^{i}$ and $\Psi_{S}(4.1)-(4.4)$ the $u_{R^{-}}$quark channel in the quark sector, the effective Lagrangian reads

$$
\begin{aligned}
\mathcal{L}= & \sum_{i} \bar{\Psi}_{D}^{i}\left(i \gamma^{\mu} \partial_{\mu}-M_{D}^{i}\right) \Psi_{D}^{i}+\bar{\Psi}_{S}\left(i \gamma^{\mu} \partial_{\mu}-M_{S}\right) \Psi_{S} \\
& -e \sum_{i} Q_{i}\left(\bar{\Psi}_{D}^{i} \gamma^{\mu} \Psi_{D}^{i}\right) A_{\mu}-(2 / 3) e\left(\bar{\Psi}_{S} \gamma^{\mu} \Psi_{S}\right) A_{\mu} \\
& -\frac{g_{2}}{\sqrt{2}} \sum_{i} \bar{\Psi}_{D}^{i} \gamma^{\mu}\left(T^{+} W_{\mu}^{+}+T^{-} W_{\mu}^{-}\right) \Psi_{D}^{i} \\
& -\frac{g_{2}}{2 \cos \theta_{W}}\left\{\sum_{i} \bar{\Psi}_{D}^{i} \gamma^{\mu}\left(g_{V}^{i}+g_{A}^{i}\right) \Psi_{D}^{i}+\bar{\Psi}_{S} \gamma^{\mu}\left(g_{V}^{1}-g_{A}^{1}\right) \Psi_{S}\right\} Z_{\mu}
\end{aligned}
$$

\footnotetext{
${ }^{6}$ Calculations can be found in refs. [16-18] and their appendices for the simple case of the four-fermion operator (3.8), where composite particles carry the definite quantum numbers of the $S U_{L}(2)$-gauge symmetry, and their tree-level gauge-couplings to the $S U_{L}(2)$-gauge bosons can be obtained by the Ward identity associating to the $S U_{L}(2)$-gauge symmetry in the UV-domain of the symmetric phase.
} 
where $i$ remains as the $S U_{L}(2)$-isospin index. For the $d_{R}$-quark channel, the composite particles made by $u_{R a} \rightarrow d_{R a}$, the coupling $g_{V, A}^{1} \rightarrow g_{V, A}^{2}$ and $(2 / 3) e \rightarrow(-1 / 3) e$. These massive composite fermions couple to the gauge bosons $W_{\mu}^{ \pm}=\left(W_{\mu}^{1} \mp i W_{\mu}^{2}\right) / \sqrt{2}$, $A_{\mu}=B_{\mu} \cos \theta_{W}+W_{\mu}^{3} \sin \theta_{W}$ and $Z_{\mu}=-B_{\mu} \sin \theta_{W}+W_{\mu}^{3} \cos \theta_{W}$. The Lagrangian (4.8) shows that the massive spectrum and interacting vertex are vector-like fully preserving the parity symmetry. Namely, each left-handed (right-handed) SM elementary fermion has its counterpart of right-handed (left-handed) composite fermion with the same quantum numbers of the SM chiral gauge symmetries. They form a massive Dirac composite fermion vectorially coupling to the SM gauge bosons.

For the massive composite bosons $S U_{L}(2)$-doublet $\mathcal{A}^{i}$ (4.1) for the $u_{R}$ channel, i.e., the neutral component $\mathcal{A}^{1} \sim\left(\bar{u}_{R a} u_{L}^{a}\right)$ and charged component $\mathcal{A}^{2} \sim\left(\bar{u}_{R a} d_{L}^{a}\right)$ of the $U_{Y}(1)$ hypercharge $Y=-1 / 2$, the effective Lagrangian reads

$$
\mathcal{L}=\sum_{i}\left[\left|\mathcal{D} \mathcal{A}^{i}\right|^{2}+M_{\Pi}^{2}\left|\mathcal{A}^{i}\right|^{2}\right]
$$

where

$$
\begin{aligned}
\mathcal{D} \mathcal{A}^{i} & =\left(\partial_{\mu}+i g_{2} \sigma \cdot \mathbf{W}_{\mu} / 2+i g_{1} Y B_{\mu}\right) \mathcal{A}^{i} \\
& =\left[\partial_{\mu}+i \frac{g_{2}}{\sqrt{2}}\left(T^{+} W_{\mu}^{+}+T^{-} W_{\mu}^{-}\right)+i e\left(\begin{array}{cc}
\sin ^{-1} 2 \theta_{W} Z_{\mu} & 0 \\
0 & -\tan ^{-1} 2 \theta_{W} Z_{\mu}-A_{\mu}
\end{array}\right)\right] \mathcal{A}^{i}
\end{aligned}
$$

and $\sigma$ is the Pauli matrix in the isospin space. Instead, for the massive composite bosons $S U_{L}(2)$-doublet $\mathcal{A}^{i}$ for the $d_{R}$ channel, i.e., the charged component $\mathcal{A}^{1} \sim\left(\bar{d}_{R a} u_{L}^{a}\right)$ and neutral component $\mathcal{A}^{2} \sim\left(\bar{d}_{R a} d_{L}^{a}\right)$ of the $U_{Y}(1)$-hypercharge $Y=1 / 2$, Equation (4.10) becomes

$$
\begin{aligned}
\mathcal{D} \mathcal{A}^{i} & =\left(\partial_{\mu}+i g_{2} \sigma \cdot \mathbf{W}_{\mu} / 2+i g_{1} Y B_{\mu}\right) \mathcal{A}^{i} \\
& =\left[\partial_{\mu}+i \frac{g_{2}}{\sqrt{2}}\left(T^{+} W_{\mu}^{+}+T^{-} W_{\mu}^{-}\right)+i e\left(\begin{array}{cc}
\tan ^{-1} 2 \theta_{W} Z_{\mu}+A_{\mu} & 0 \\
0 & -\sin ^{-1} 2 \theta_{W} Z_{\mu}
\end{array}\right)\right] \mathcal{A}^{i}
\end{aligned}
$$

The last matrix-terms in eqs. (4.10) and (4.11) respectively show that the isospin components $\mathcal{A}^{1} \sim \bar{u}_{R} u_{L}$ and $\mathcal{A}^{2} \sim \bar{d}_{R} d_{L}$ have no electric charge, but the $U_{Y}(1)$ hypercharge coupling to $Z^{0}$. The gauge couplings in the effective Lagrangian (4.8), (4.10) and (4.11) are consistent with SM gauge bosons couplings to the elementary fermions inside the composite particles, see figure 3.

The effective Lagrangian (4.8), (4.10) and (4.11) can be generalized to the lepton sector and lepton-quark sector. Note that in the effective Lagrangian (4.8) and (4.9), we only present the spectra (kinetic terms or propagators) of massive composite particles and their tree-level gauge-coupling to the elementary SM gauge bosons.

To the leading order of the expansion in the powers of the perturbative SM gauge couplings (the tree-level), the interacting vertexes of composite bosons and fermions coupling to SM gauge bosons $\left(\gamma, W^{ \pm}, Z^{0}\right)$ are represented by the tree-level Feynman diagrams, see figure 3 , which show gauge bosons interacting with one of elementary particles inside 

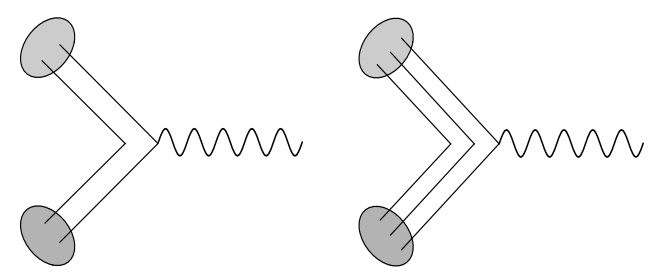

Figure 3. We sketch the vertexes of composite boson (left) and fermion (right) interacting with SM gauge bosons (wave line), and the SM elementary fermion is represented by a solid line. In general, these vertexes should be understood as one-particle-irreducible vertexes of SM gauge bosons interacting with the entire composite particle as an elementary particle. At the lowest tree-level order of perturbative SM gauge couplings, the vertexes can be represented by the sum of possible gauge couplings to an SM elementary particle inside a composite particle. Note that we do not show the vertexes of composite boson (left) interacting with two SM gauge bosons that are present in the effective Lagrangian (4.9).

composite particles. Therefore, this implies that at the tree level, some calculations of Feynman diagrams are similar to those in the SM, however, the fermion spectra and coupling vertexes are vectorlike, see eqs. (4.7) and (4.8). The final results are characterized by the mass scales $M_{\Pi, F}(4.4)$ of composite fermions

$$
M_{F}>M_{\Pi} \propto \mathcal{E}_{\xi}
$$

in the UV domain and functions of the SM gauge couplings $g_{1}, g_{2}, g_{3}$ at this mass scale $M_{\Pi, F}$, rather than the electroweak scale $v \approx 239.5 \mathrm{GeV}$ in the IR domain.

Since the propagators of massive composite particles are obtained from the lowest nontrivial contribution of the strong-coupling expansion [14, 15], it cannot be precluded that there could be the interacting vertexes between massive composite particles, e.g., the Yukawa-type interactions of composite bosons and fermions, stemming from the high-order contributions of the strong-coupling expansion. In this article, we do not attempt to discuss the interacting vertexes among composite particles and their relevance in the UV-domain.

\subsubsection{Some discussions on FCNC and anomalies in UV-domain}

We attempt to have some discussions on the analogical FCNC processes of the UV effective Lagrangian (4.8) and (4.9) of composite particles in the UV-domain. The compositefermion case is considered as an example for discussions that apply also to the compositeboson case. Recall that (i) composite fermions $\Psi^{f}$ are composed by the SM elementary fermions $\psi^{f}$ in the same family " $f$ "; (ii) the fermion-family "diagonalized" effective Lagrangian (4.8), (4.9) of massive composite particles $\Psi^{f}$ is obtained from the fermion-family "diagonalized" four-fermion operators (2.10) in the mass eigenstates of the SM elementary fermions $\psi^{f}$; (iii) the mass eigenstates of composite fermions coincide with the mass eigenstates of the SM elementary fermions in the scenes that composite fermions are composed by elementary fermions in their mass eigenstates, see section 4.1; (iv) the mass and gauge eigenstates of composite fermions are identical, due to the exact conservation of the SM chiral gauge symmetries. 
In the representation of mass eigenstates of composite fermions, totally contrary to the IR effective Lagrangian for the SM in the IR domain, see section 3.1 and in particular section 3.1.3, the UV effective Lagrangian (4.8) and (4.9) are realized in the scaling UV-domain of the symmetric phase, where the expectational values of two-fermion operators $(2.11)\left\langle\psi_{R}^{f} \bar{\psi}_{L}^{f}\right\rangle \propto m^{f} \equiv 0, f=1,2,3$, identically vanish. The strong-coupling dynamics that forms massive composite particles in the symmetric phase is totally independent of the SM elementary fermion masses $m_{f}$ in the symmetry-breaking phase. The SM chiral-gauge symmetries and global fermion-family symmetry are exactly preserved by the strong-coupling dynamics and massive spectrum of composite fermions. More precisely, in the representation of mass eigenstates of composite fermions, the fermion-family number associating to the symmetry $U_{L}^{f}(1) \times U_{R}^{f}(1) f=1,2,3$ for the composite fermion $\Psi^{f}$ in each family is exactly conserved. Therefore, any process violating fermion-family number is impossible, namely any $\mathcal{F} C N C$ process is completely prohibited. Here the notation $\mathcal{F} C N C$ refers to the process of changing one composite fermion $\Psi^{f}$ flavor to another composite fermion $\Psi^{f^{\prime}}$ flavor with the same electric charge, which is different from the notion FCNC of the IR effective Lagrangian for the SM elementary fermions in the IR-domain.

However, as perturbatively turning on chiral-gauge-boson interactions to composite fermions, does the interactions between the chiral-gauge-boson $W^{ \pm}$and composite fermions introduce a suppressed 1PI vertex for the $\mathcal{F} C N C$ at loop level, analogous to that of the SM effective Lagrangian in the IR domain? The answer is no, for the reason that the mass and gauge eigenstates of composite fermions are identical, and fermion-family mixing matrices (2.14) do not associate to the interacting vertexes of the $W^{ \pm}$boson and composite fermions (4.8). In conclusion, fermion-family-number violating processes like $\mathcal{F} C N C$ are impossible for the effective Lagrangian (4.8) and (4.9) in the UV-domain. The remained question is how the suppressed 1PI vertexes of the FCNC from the SM $W^{ \pm}$ boson loop and CKM-type mixing matrices in the IR-domain is induced, when composite fermions decay into SM elementary fermions, a phase transition occurs from symmetric phase $\left(m_{f}=0\right)$ to symmetry-breaking phase $\left(m_{f} \neq 0\right)$. This is deserved to study in another article.

In the SM Lagrangian (4.7) of chiral-gauge symmetries and chiral-gauged fermion spectra, the chiral-gauge anomalies associating to the $S U_{L}(2) \times U_{Y}(1)$ chiral-gauge symmetries are canceled, because of the $S U_{L}(2)$ Lie algebra and the SM fermion content of quarks and leptons in each fermion family, and the non conservation of fermion number $(B-L)$ is given by the instanton contribution. In the effective Lagrangian (4.8) of composite particles, although the gauge symmetries are still chiral, i.e., $S U_{L}(2) \times U_{Y}(1)$, the spectra of composite fermions $\Psi_{D}$ and $\Psi_{S}$ are vector-likely gauged. As a result, the chiral-gauge anomalies from the left-handed composite fermions are exactly canceled by the anomalies from their right-handed counterparts. In the effective Lagrangian (4.8) of composite fermions, we obtained [16] the non conservation of fermion number $(B-L)$ by considering the mixing anomaly [65].

As the running energy scale $\mu$ decreasing, and composite particle's form factor and binding energy vanishing, the composite particles become unstable and dissolve (decay) to their constitutes of SM elementary particles as final states. In the following, we discuss 
the decay and annihilation channels of the composite particles into the SM elementary particles, in particular two gauge bosons, as final states.

\section{Composite pesudo scalar bosons decay}

This section 5 and next section 6 discuss the decays and other relevant processes of composite bosons and fermions interacting with SM gauge bosons described by the effective Lagrangians (4.8) and (4.9) of the effective theory of composite particles in UV-domain. These results provide possible connections with the diboson and other channels in ATLAS and CMS experiments.

\subsection{Low-energy QCD pions and their decays}

First let us recall the low-energy hadronic physics of mesons (baryons) that are the bound states of two quarks (three quarks) due to the confinement dynamics of the local SU(3)color gauge theory (QCD). This can be approximately described by the effective low-energy Lagrangian of baryons and mesons, which could be realized in the possible low-energy IRdomain of the QCD theory. On the other hand, asymptotically-free states of quarks and gluons are realized in the high-energy UV-domain of the QCD theory. In both bound states and asymptotically-free states, elementary quarks have the same electroweak couplings to the SM gauge bosons $W^{ \pm}, Z^{0}$ and $\gamma$.

To effectively study the non-perturbative nature of low-energy QCD theory, the global chiral-symmetric Lagrangian ( $\sigma$-model) was adopted to describe the massless QCDbound states of elementary $u$ and $d$ quarks: proton $p(u u d)$, neutron $n(d d u)$ and pions $\pi^{0, \pm}$ (uu,dd,ud). The spontaneous breaking of the $S U_{L}(2) \times S U_{R}(2)$ chiral symmetries leads to the massive doublet baryon fields $(p, n)$ and massless triplet pion fields $\pi^{k}$ as three Goldstone bosons. The explicit breaking induced by the $u$ and $d$ quark masses leads to massive pion fields $\pi^{k}$ and the partial conservation of the axial current (PCAC)

$$
\partial^{\mu} A_{\mu}^{j}(x)=m_{\pi}^{2} f_{\pi} \pi^{j}(x)
$$

where the quark doublet $\psi^{i}=(u, d)$ and the axial current $A_{\mu}^{j}(x)=\bar{\psi} \gamma_{\mu} \gamma_{5}\left(\sigma^{j} / 2\right) \psi$. The latter couples to the pion fields $\pi^{j}(x)$ due to the spontaneous breaking of chiral symmetries, leading to the nontrivial matrix elements between the pion state $\left|\pi^{k}(p)\right\rangle$ and the vacuum

$$
\left\langle 0\left|A_{\mu}^{j}(x)\right| \pi^{k}(p)\right\rangle=i p_{\mu} f_{\pi} \delta^{j k} e^{-i p x}, \quad\left\langle 0\left|\partial^{\mu} A_{\mu}^{j}(x)\right| \pi^{k}(p)\right\rangle=m_{\pi}^{2} f_{\pi} \delta^{j k} e^{-i p x} .
$$

The first matrix element defines the pion decay constant (form factor) $f_{\pi}$, the second matrix element and the mass-shell condition $p^{2}=m_{\pi}^{2}$ defines the pion mass $m_{\pi}$. The charged pions $\pi^{ \pm}=\left(\pi^{1} \mp i \pi^{2}\right) / 2=\bar{d} \gamma_{5} u, \bar{u} \gamma_{5} d$ and neutral pion $\pi^{0}=\pi^{3} / 2=\left(\bar{u} \gamma_{5} u-\bar{d} \gamma_{5} d\right) / 2$. For the isospin $j, k=1,2$ components in eq. (5.1), the first matrix element determines the rate of the decay $\pi^{+}(\bar{u} d) \rightarrow \mu^{+}+\nu_{\mu}$ and experimental value $f_{\pi} \approx 93 \mathrm{MeV}$. In addition, for the isospin $j=k=3$ component in eq. (5.1) reads

$$
\partial^{\mu} A_{\mu}^{3}=m_{\pi}^{2} f_{\pi} \pi^{0}-\frac{\alpha}{8 \pi} \epsilon_{\mu \nu \rho \sigma} F^{\mu \nu} F^{\rho \sigma},
$$




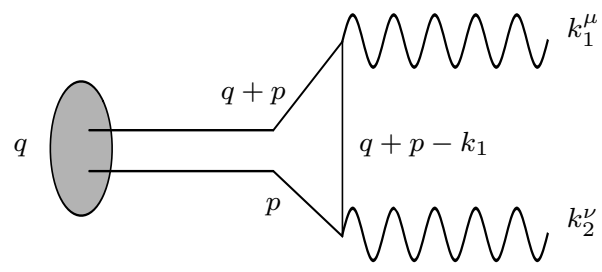

Figure 4. We show the triangle diagram (a quark loop of solid line) of a composite boson (two parallel solid lines associating to a pseudo-scalar vertex $\gamma_{5}$ ) decaying into the two SM gauge bosons (two wave lines associating to two vector-like vertexes $\gamma_{\mu}$ and $\gamma_{\nu}$ ).

receiving an axial anomaly in terms of the gauge field (photon) strength $F$ and finestructure constant $\alpha$, contributed from the triangle diagram shown in figure 4 . This axial anomaly dominates the $\pi^{0}$-decay rate,

$$
\Gamma_{\pi^{0} \rightarrow \gamma \gamma}=\left(\frac{\alpha N_{c}}{3 \pi f_{\pi}}\right)^{2} \frac{m_{\pi^{0}}^{3}}{64 \pi}
$$

in excellent agreement with the experimental value.

\subsection{Scalar and pesudo scalar composite bosons}

We attempt to study the decays of composite bosons $\mathcal{A}^{i}$ in the UV-domain, discussed in sections 4.1 and 4.2. For the sake of simplicity, we adopt the first quark family $\psi_{L}^{i}=(u, d)_{L}$ and $\psi_{R}=u_{R}, d_{R}$ for illustrations. For the $u$-channel, the massive composite boson $\mathcal{A}^{i}=$ $\left(\bar{u}_{R} \psi_{L}^{i}\right)$ (4.1), which is an $S U_{L}(2)$ doublet and $U_{Y}(1)$ charged $Y=-1 / 2$, can expressed in terms of scalar and pseudo scalar fields,

$$
\mathcal{A}^{i}=\left(\bar{u}_{R} \psi_{L}^{i}\right)=(1 / 2)\left[\left(\bar{u} \psi^{i}\right)-\left(\bar{u} \gamma_{5} \psi^{i}\right)\right] .
$$

where the form factor $\left[Z_{\Pi}^{S}\right]^{-1 / 2}$ is omitted for simplifying notations. There are four components

$$
S^{0}=\bar{u} u, \quad S^{-}=\bar{u} d ; \quad \Pi^{0}=\bar{u} \gamma_{5} u, \quad \Pi^{-}=\bar{u} \gamma_{5} d
$$

where the isospin index $i=1,2$ are relabeled as $i^{\prime}=0, \pm$ indicating the neutral or charged component. For the $d$-channel, by the substitution $u \rightarrow d$ in eqs. (5.5) and (5.6), we have

$$
\begin{aligned}
& \mathcal{A}^{i}=\left(\bar{d}_{R} \psi_{L}^{i}\right)=(1 / 2)\left[\left(\bar{d} \psi^{i}\right)-\left(\bar{d} \gamma_{5} \psi^{i}\right)\right], \\
& S^{0}=\bar{d} d, \quad S^{+}=\bar{d} u ; \quad \Pi^{0}=\bar{d} \gamma_{5} d, \quad \Pi^{+}=\bar{d} \gamma_{5} u .
\end{aligned}
$$

The neutral state $\Pi^{0}$ has the contributions from eqs. (5.6) and (5.7) that can be mixed up, but is different from the normal pion $\pi^{0}=(\bar{u} u-\bar{d} d) / \sqrt{2}$ in the SU(3) quark model where the minus sign comes from the isospin $\tau^{3}=(1,-1)$. The discussions can be generalized to other families including leptons. The second and third families of composite bosons are simply replicated by the replacements $u \rightarrow c, t$ and $d \rightarrow s, b$. There is no mixing among three families of composite bosons. The gauge interactions at the leading order (tree-level) of gauge couplings (4.10) and (4.11) do not introduce the mixing of composite bosons (5.6) with its counterparts in the second or third family. 
These composite bosons $\Pi^{0, \pm}(5.6)$ and (5.7) are bound by the strong-coupling dynamics in the UV domain, differently from the QCD-bound mesons. They have the same chiral-invariant mass $M_{\Pi}$ (4.4). Instead the three pion fields $\pi^{0, \pm}$ have the chiral-variant mass $m_{\pi}$. The vectorial and axial currents associating to the global $S U_{L}(2) \times U_{Y}(1)$ chiral symmetries are exactly conserved because these chiral symmetries are preserved, apart from the chiral gauge anomaly (axial anomaly). Instead the axial current associating to the global chiral symmetries of the effective low-energy Lagrangian of the QCD-bound states are partially conserved because of spontaneous and explicit chiral-symmetry-breaking (PCAC). However, the massive pseudo scalar fields $\Pi^{0, \pm}$ (5.6), (5.7) and the QCD pion fields $\pi^{0, \pm}$ have the same quantum numbers of the SM symmetries. Moreover, we shall show the pseudo-scalar fields $\Pi^{0, \pm}$ decay into SM elementray particles, in particular the $\Pi^{0}$-decay into two SM gauge bosons, analogously to the decay $\pi^{0} \rightarrow \gamma \gamma$, attributing to the axial anomaly (5.4).

\subsection{Composite particle $\Pi^{0}$ decay into two $\mathrm{SM}$ gauge bosons}

\subsection{1 $\Pi^{0}$ decay into two photons}

Suppose the vacuum state is $|\mathbf{0}\rangle$ in the UV domain, the composite-boson momentum state $\left|\mathcal{A}^{i}(q)\right\rangle$ on the mass shell can be defined by the field operator $\mathcal{A}^{i}(q)$ or $\mathcal{A}^{i}(x)$ as follow

$$
\left|\mathcal{A}^{i}(q)\right\rangle \equiv \frac{1}{F_{\Pi}} \mathcal{A}^{i \dagger}(q)|\mathbf{0}\rangle=\frac{1}{F_{\Pi}} \int d^{4} x e^{-i q x} \mathcal{A}^{i \dagger}(x)|\mathbf{0}\rangle,
$$

with the normalization

$$
\left\langle\mathcal{A}^{i}(q) \mid \mathcal{A}^{i^{\prime}}\left(q^{\prime}\right)\right\rangle=\delta^{i i^{\prime}}(2 \pi)^{4} \delta^{4}\left(q-q^{\prime}\right)
$$

or equivalently

$$
\left\langle\mathbf{0}\left|\mathcal{A}^{i}(x)\right| \mathcal{A}^{i^{\prime}}(q)\right\rangle=F_{\Pi} \delta^{i i^{\prime}} e^{i q x},
$$

where $F_{\Pi}^{-1}=F_{\Pi}^{-1}\left(q^{2}\right)$ relates to the form factor $\left[Z_{\Pi}^{S}\right]^{-1 / 2}(4.1)$, as discussed in section 4 . As will be seen soon, $F_{\Pi}$ is in fact the composite-boson decay constant. From eq. (5.10), the matrix element reads

$$
\left\langle\mathbf{0}\left|\partial^{\mu} \mathcal{A}^{i}(x)\right| \mathcal{A}^{i^{\prime}}(q)\right\rangle=i q^{\mu} F_{\Pi} \delta^{i i^{\prime}} e^{i q x} .
$$

The effective Lagrangian (4.9) gives the equation of motion and the mass-shell condition $q^{2}=M_{\Pi}^{2}$ of the composite boson $\mathcal{A}^{i}(x)$. From eq. (5.11), we have

$$
\left\langle\mathbf{0}\left|\left(\partial^{\mu}\right)^{2} \mathcal{A}^{i}(x)\right| \mathcal{A}^{i^{\prime}}(q)\right\rangle=M_{\Pi}^{2} F_{\Pi} \delta^{i i^{\prime}} e^{i q x} .
$$

The matrix element (5.10) relates to the composite-boson propagator,

$$
\left\langle\mathbf{0}\left|\mathcal{A}^{i}(0)\right| \mathcal{A}^{i^{\prime}}(q)\right\rangle=\frac{1}{F_{\Pi}} \int d^{4} x\left\langle\mathbf{0}\left|\mathcal{A}^{i}(0) \mathcal{A}^{i^{\prime} \dagger}(x)\right| \mathbf{0}\right\rangle e^{-i q x}=\frac{\delta^{i i^{\prime}} F_{\Pi}^{-1}\left(q^{2}\right)}{q^{2}-M_{\Pi}^{2}} .
$$

The matrix element of composite particle $\Pi^{0}$ in eqs. (5.6) and (5.7) reads,

$$
\left\langle f\left(p^{\prime}\right)\left|\Pi^{0}(0)\right| f(p)\right\rangle=\frac{g_{\Pi^{0}}}{-q^{2}+M_{\Pi}^{2}} \bar{u}_{f}\left(p^{\prime}\right) \gamma_{5} u_{f}(p), \quad q=p^{\prime}-p
$$


where the $g_{\Pi^{0}}=g_{\Pi^{0}}\left(q^{2}\right)$ is the $\Pi^{0}$-coupling to its constituent fermions $f=u, d$ quarks of mass $m_{f}$ and wave function $u_{f}$. Actually, the coupling $g_{\Pi^{0}}\left(q^{2}\right)$ is the 1 PI vertex function of composite particle $\Pi^{0}$ and its constituent fermions and we parametrize it by the form factor $F_{\Pi}^{-1}\left(q^{2}\right)$ and constituent mass $m_{f}$

$$
g_{\Pi^{0}}\left(q^{2}\right)=m_{f} F_{\Pi}^{-1}\left(q^{2}\right),
$$

and $g_{\Pi^{0}}$ is finite as $m_{f} \rightarrow 0$, see for example [66]. These definitions and equations apply also for scalar composite bosons $S^{i}$ and pseudo scalar composite bosons $\Pi^{i}$, see eqs. (5.6) and (5.7).

We are in the position of discussing the rate of the neutral $\Pi^{0}$ decay into two SM gauge bosons, i.e., diboson channels:

$$
\Pi^{0} \rightarrow G+G^{\prime},
$$

where $G$ and $G^{\prime}$ represent the SM gauge bosons $W^{ \pm}, Z^{0}$ and $\gamma$. The amplitude of the decay (5.16) is defined by the matrix element

$$
\left.\mathcal{F}\left(q^{2}\right)\right|_{q^{2} \rightarrow M_{\Pi^{0}}^{2}}=\left.\epsilon_{1}^{\mu}\left(k_{1}\right) \epsilon_{2}^{\nu}\left(k_{2}\right) T_{\mu \nu}\left(k_{1}, k_{2}, q\right)\right|_{q^{2} \rightarrow M_{\Pi^{0}}^{2}}, \quad q=k_{1}+k_{2}
$$

and

$$
T_{\mu \nu}\left(k_{1}, k_{2}, q\right)=\left(q^{2}-M_{\Pi^{0}}^{2}\right)\left(g g^{\prime}\right) \int d^{4} x d^{4} y e^{i k_{1} x+i k_{2} y}\left\langle\mathbf{0}\left|T\left(J_{\mu}(z) J_{\nu}^{\prime}(y) \Pi^{0}(0)\right)\right| \mathbf{0}\right\rangle,
$$

where $k_{1,2}$ and $\epsilon_{1,2}$ are four momenta and polarizations of the SM gauge bosons $G$ and $G^{\prime}$, and $J_{\mu}(z)\left[J_{\nu}^{\prime}(y)\right]$ is fermion current coupling $g\left[g^{\prime}\right]$ to the SM gauge boson $G\left[G^{\prime}\right]$. The amplitude $T_{\mu \nu}\left(k_{1}, k_{2}, q\right)$ has the symmetry $\left(k_{1}, \mu\right) \leftrightarrow\left(k_{2}, \nu\right)$.

The lowest one-loop contribution to the vertex function (5.18) is represented by the triangle Feynman diagram, see figure 4. Considering eqs. (5.14) and (5.15), the $\Pi^{0}$-decay amplitude $T_{\mu \nu}$ (5.18) computed at this one-loop level is given by

$$
\begin{aligned}
T_{\mu \nu}\left(k_{1}, k_{2}, q\right)= & T_{\mu \nu}^{(1)}\left(k_{1}, k_{2}, q, m_{f}\right)+T_{\nu \mu}^{(2)}\left(k_{2}, k_{1}, q, m_{f}\right), \\
T_{\mu \nu}^{(1)}\left(k_{1}, k_{2}, q, m_{f}\right)= & -\left(g g^{\prime}\right) N_{c} m_{f} F_{\Pi}^{-1}\left(q^{2}\right) \times \\
& \times \int \frac{d^{4} p}{(2 \pi)^{4}} \frac{\operatorname{tr}\left[\left(\not q+\not p-\not k_{1}+m_{f}\right) \Gamma_{\mu}\left(\not q+\not p+m_{f}\right) \gamma_{5}\left(\not p+m_{f}\right) \Gamma_{\nu}^{\prime}\right]}{\left[\left(q+p-k_{1}\right)^{2}-m_{f}^{2}\right]\left[(q+p)^{2}-m_{f}^{2}\right]\left(p^{2}-m_{f}^{2}\right)},
\end{aligned}
$$

where the coupling vertexes $g \Gamma_{\mu}$ and $g^{\prime} \Gamma_{\nu}^{\prime}$ to the SM gauge bosons $G$ and $G^{\prime}$ are given by the SM Lagrangian (4.8), and the trace "tr" is over the spinor space. Equation (5.20) is not well-defined because of the linear divergence of the momentum integral. Introducing the Pauli-Villars mass $\mathcal{M}$ that plays the role of the UV cutoff $\Lambda$, we adopt the Pauli-Villars regularization,

$$
T_{\mu \nu}^{(1)}\left(k_{1}, k_{2}, q, m_{f}\right) \rightarrow T_{\mu \nu}^{(1)}\left(k_{1}, k_{2}, q, m_{f}\right)-T_{\mu \nu}^{(1)}\left(k_{1}, k_{2}, q, \mathcal{M}\right)
$$

to make the momentum integral to be finite and well-defined. Note that the Pauli-Villas regularization $\mathcal{M}$ explicitly breaks chiral gauge symmetries of effective Lagrangian. 
For the process $\Pi^{0} \rightarrow \gamma+\gamma, g=g^{\prime}=e Q^{i}, \Gamma_{\mu}=\gamma_{\mu}$ and $\Gamma_{\nu}^{\prime}=\gamma_{\nu}$ in eq. (5.20). The $J_{\mu}(z)$ and $J_{\nu}^{\prime}(y)$ in eq. (5.18) are vector-like and conserved fermion currents coupling with two on-shell photons $k_{1}$ and $k_{2}$. The trace in the numerator of eq. (5.20) is equal to $4 i m_{f} \epsilon_{\mu \nu \rho \sigma} k_{1}^{\sigma} k_{2}^{\rho}$. With the on-shell conditions $k_{1}^{2}=0, k_{2}^{2}=0$ and $2 k_{1} \cdot k_{2}=q^{2}$, the two contributions (5.19) to the amplitude (5.17) of $\Pi^{0}$ decaying into two photons is given by

$$
\begin{aligned}
\left.\mathcal{F}\left(q^{2}\right)\right|_{q^{2} \rightarrow M_{\Pi^{0}}^{2}}= & \frac{\left(g g^{\prime}\right) N_{c}}{2 \pi^{2} F_{\Pi}} \epsilon_{\mu \nu \rho \sigma} \epsilon_{1}^{\mu} \epsilon_{2}^{\nu} k_{1}^{\rho} k_{2}^{\sigma} \\
& \times \int_{0}^{1} d x \int_{0}^{1-x} d y\left[\frac{\mathcal{M}^{2}}{\mathcal{M}^{2}-x y q^{2}}-\frac{m_{f}^{2}}{m_{f}^{2}-x y q^{2}}\right]_{q^{2} \rightarrow M_{\Pi^{0}}^{2}} \\
= & \frac{\left(g g^{\prime}\right) N_{c}}{4 \pi^{2} F_{\Pi}} \epsilon_{\mu \nu \rho \sigma} \epsilon_{1}^{\mu} \epsilon_{2}^{\nu} k_{1}^{\rho} k_{2}^{\sigma} .
\end{aligned}
$$

The second term in eq. (5.22) is suppressed for $M_{\Pi^{0}} \gg m_{f}$ and the first term for $\mathcal{M} \rightarrow \infty$ leading to the final result (5.23). The result is just the axial anomaly of the triangle Feynman diagram (figure 4 ) with one axial vertex " $\gamma_{5}$ " and two vector-like (AVV) vertexes " $\gamma_{5} \gamma_{\mu} \gamma_{\nu}$ ". This axial-anomaly result (5.23) is due to the regularization that does not preserve chiral gauge symmetries of effective Lagrangian, and it is independent of highorder contributions, constituent fermion mass $m_{f}$ and UV cutoff $\mathcal{M}$. Equation (5.23) shows that the $F_{\Pi}$ value at $q^{2}=M_{\Pi^{0}}^{2}$ is the $\Pi^{0}$-decay constant. For the first quark family, there are two $\Pi^{0}$ channels (5.6) and (5.7) with $Q_{i}=(2 / 3,-1 / 3)$, i.e., $g^{2}=e^{2}(4 / 9,1 / 9)$. The total amplitude of $\Pi^{0} \rightarrow \gamma+\gamma$ reads,

$$
\begin{aligned}
\left.\mathcal{F}\left(q^{2}\right)\right|_{q^{2} \rightarrow M_{\Pi^{0}}^{2}} ^{\Pi^{0} \rightarrow \gamma+\gamma} & =\sum_{i}\left(Q^{i}\right)^{2} \frac{e^{2} N_{c}}{4 \pi^{2} F_{\Pi}} \epsilon_{\mu \nu \rho \sigma} \epsilon_{1}^{\mu} \epsilon_{2}^{\nu} k_{1}^{\rho} k_{2}^{\sigma}=\left(\frac{5}{9}\right) \mathcal{F} \\
\mathcal{F} & \equiv \frac{e^{2} N_{c}}{4 \pi^{2} F_{\Pi}} \epsilon_{\mu \nu \rho \sigma} \epsilon_{1}^{\mu} \epsilon_{2}^{\nu} k_{1}^{\rho} k_{2}^{\sigma} .
\end{aligned}
$$

The amplitude (5.24) should be multiplied by a factor $N_{c}=3$, if we consider three quark families.

It needs some more clarifications on the amplitude (5.24). Both the composite pseudoscalar boson $\Pi^{0}$ and the composite scalar Higgs boson $H$ decay into the two-photon state, however these two channels are not mixed. They have different parities, masses (invariant masses) and decay constants. The former composed by the strong-coupling dynamics in the UV-domain decay into two photons via the pseudo-scalar coupling (see figure 4) to the loop of $u$ and $d$ quarks. Whereas the latter composed by the NJL-dynamics in the IR-domain via the scalar coupling $\bar{t} t H$ (4.7) to a top-quark loop. In the LHC $p p$ collision, the first family of composite particles made of $u$ and $d$ quarks are more probably produced, compared with other families of composite particles that are produced by small SM gauge interacting vertexes.

\subsection{2 $\Pi^{0}$ decay into $\gamma+Z^{0}, W^{+}+W^{-}$and $Z^{0}+Z^{0}$}

Let us study other possible decay channels $\Pi^{0} \rightarrow \gamma+Z^{0}, \Pi^{0} \rightarrow W^{+}+W^{-}$and $\Pi^{0} \rightarrow$ $Z^{0}+Z^{0}$. Suppose that the pseudo scalar composite field $\Pi^{0}(x)$ is heavy and its mass $M_{\Pi^{0}}$ 
is much larger than the $W^{ \pm}$and $Z^{0}$ masses, the intermediate gauge bosons $W^{ \pm}$and $Z^{0}$ are considered to be approximately massless, $k_{1}^{2} \approx 0, k_{2}^{2} \approx 0$ and $2 k_{1} \cdot k_{2} \approx q^{2}$. In addition, the coupling-vertexes $g \Gamma_{\mu}$ and $g^{\prime} \Gamma_{\nu}^{\prime}$ in eq. (5.20) are given in the SM Lagrangian (4.7), they contain both axial and vector-like vertexes. We consider the vector-like vertexes so that the triangle Feynman diagram (figure 4) and the amplitude (5.18) has the AVV structure, whose nontrivial axial-anomaly amplitude can be compared with the amplitude (5.24) of the channel $\Pi^{0} \rightarrow \gamma+\gamma$. As a result, for the process $\Pi^{0} \rightarrow \gamma+Z^{0}$, the couplings $g=e Q^{i}$ and $g^{\prime}=g_{2} g_{V}^{i} /\left(2 \cos \theta_{W}\right)$, we obtain from eq. (5.23) the amplitude,

$$
\begin{aligned}
\left.\mathcal{F}\left(q^{2}\right)\right|_{q^{2} \rightarrow M_{\Pi^{0}}^{2}} ^{\Pi^{0} \rightarrow \gamma+Z^{0}} & =\frac{1}{\sin 2 \theta_{W}} \sum_{i}\left(Q^{i} g_{V}^{i}\right) \mathcal{F} \\
& =\frac{1}{\sin 2 \theta_{W}}\left(\frac{1}{2}-\frac{5}{9} \sin \theta_{W}^{2}\right) \mathcal{F} .
\end{aligned}
$$

For the process $\Pi^{0} \rightarrow W^{+}+W^{-}$, the couplings $g=g^{\prime}=g_{2} /(2 \sqrt{2})$ and we obtain from eq. (5.23) the amplitude,

$$
\left.\mathcal{F}\left(q^{2}\right)\right|_{q^{2} \rightarrow M_{\Pi^{0}}^{2}} ^{\Pi^{0} \rightarrow W^{+}+W^{-}}=\frac{1}{8 \sin ^{2} \theta_{W}} \sum_{i} \mathcal{F}=\frac{1}{8 \sin ^{2} \theta_{W}} \mathcal{F},
$$

where the sum over isospin " $i$ " gives one in this case, see eq. (4.7). For the process $\Pi^{0} \rightarrow Z^{0}+Z^{0}$, the couplings $g=g^{\prime}=g_{2} g_{V}^{i} /\left(2 \cos \theta_{W}\right)$ and we obtain from eq. (5.23) the amplitude,

$$
\begin{aligned}
\left.\mathcal{F}\left(q^{2}\right)\right|_{q^{2} \rightarrow M_{\Pi^{0}}^{2}} ^{\Pi^{0} \rightarrow Z^{0}+Z^{0}} & =\frac{1}{\sin ^{2} 2 \theta_{W}} \sum_{i}\left(g_{V}^{i}\right)^{2} \mathcal{F} \\
& =\frac{1 / 2-\sin ^{2} \theta_{W}+(5 / 9) \sin ^{4} \theta_{W}}{\sin ^{2} 2 \theta_{W}} \mathcal{F} .
\end{aligned}
$$

Their differences from the two-photon amplitude (5.24) are only attributed to the different eletroweak couplings of the SM (4.7).

\subsection{Ratios of different channels of $\Pi^{0}$ decay into two SM gauge bosons}

The numerical rate of the neutral $\Pi^{0}(q)$ decay (5.16) at the mass shell $\left(q^{2}=M_{\Pi^{0}}^{2}\right)$ is given by integrating the squared amplitude (5.17) over the phase space of two gauge bosons,

$$
\begin{aligned}
\Gamma_{\Pi^{0} \rightarrow G+G^{\prime}}= & \frac{1}{2 M_{\Pi^{0}}} \sum_{\epsilon_{1}, \epsilon_{2}} \int \frac{d^{3} k_{1} d^{3} k_{2}}{4(2 \pi)^{6} \omega_{1} \omega_{2}}\left|\mathcal{F}\left(q^{2}\right)\right|_{\Pi^{0} \rightarrow G+G^{\prime}}^{2} \\
& \times\left.(2 \pi)^{4} \delta^{4}\left(q-k_{1}-k_{2}\right)\right|_{q^{2}=M_{\Pi}^{2}} \\
= & \mathcal{C}_{\Pi^{0} \rightarrow G+G^{\prime}} \Gamma, \\
\Gamma & \equiv\left(\frac{\alpha N_{c}}{3 \pi F_{\Pi}}\right)^{2} \frac{M_{\Pi^{0}}^{3}}{64 \pi},
\end{aligned}
$$

where the mass $M_{\Pi^{0}}$ and decay constant $F_{\Pi}$ of the composite boson $\Pi^{0}$ are totally unknown, while the coefficients $\mathcal{C}_{\Pi^{0} \rightarrow G+G^{\prime}}$ are completely determined from eqs. (5.24), (5.26), (5.27) 
and (5.28) in terms of SM gauge couplings. Note that the decay rates (5.29) depend on the $\Pi^{0}$ mass $M_{\Pi^{0}}$ and decay constant $F_{\Pi}$ determined by the strong-coupling dynamics, analogously to the pion mass $m_{\pi}$ and decay constant $f_{\pi}$ determined by the QCD dynamics.

In the rest frame of the composite meson $\Pi^{0}$, the final states of two body decay follow the energy-momentum conservations $\mathbf{p}_{1}=-\mathbf{p}_{2},\left|\mathbf{p}_{1}\right|=\left|\mathbf{p}_{2}\right|$ and have the invariant mass $\mathcal{M}_{\text {inv }}=M_{\Pi^{0}}$. The rates of the composite meson $\Pi^{0}$ decaying into two SM gauge bosons $G$ and $G^{\prime}$ are given by eq. (5.29), we then obtain the rates of diboson channel decay (5.16):

$$
\begin{aligned}
\Gamma_{\Pi^{0} \rightarrow \gamma+\gamma} & =\left(\frac{5}{9}\right)^{2} \Gamma, \\
\Gamma_{\Pi^{0} \rightarrow \gamma+Z^{0}} & =\frac{1}{\sin ^{2} 2 \theta_{W}}\left(\frac{1}{2}-\frac{5}{9} \sin ^{2} \theta_{W}\right)^{2} \Gamma, \\
\Gamma_{\Pi^{0} \rightarrow W^{+}+W^{-}} & =\left(\frac{1}{8 \sin ^{2} \theta_{W}}\right)^{2} \Gamma, \\
\Gamma_{\Pi^{0} \rightarrow Z^{0}+Z^{0}} & =\left(\frac{1 / 2-\sin ^{2} \theta_{W}+(5 / 9) \sin ^{4} \theta_{W}}{\sin ^{2} 2 \theta_{W}}\right)^{2} \Gamma .
\end{aligned}
$$

The diphoton channel (5.31) is expected to have the largest branching ratio, analogously to the QCD pion decay $\pi^{0} \rightarrow \gamma \gamma$. Suppose that the possible resonance of the diphoton channel (5.31) of the invariant mass $\mathcal{M}_{\gamma \gamma}$ is observed in high-energy experiments, it implies that the composite meson mass $M_{\Pi^{0}} \gtrsim \mathcal{M}_{\gamma \gamma}$, since there are other decay channels (5.32), (5.33), (5.34) into two gauge bosons of $\gamma Z^{0}, W^{+} W^{-}$and $Z^{0} Z^{0}$. The channel $\gamma Z^{0}$ corresponds to the final states of a photon and two leptons or a "fat" jet of two quarks. The channels $W^{+} W^{-}$and $Z^{0} Z^{0}$ correspond to the final states of (i) two "fat" jets of four quarks for $W$ and $Z$ hadronic decays or (ii) four leptons for $W$ and $Z$ leptonic decay, or a "fat" jet and two leptons in the combination of cases (i) and (ii).

For the reasons that the properties of composite particles, e.g., form-factors and masses (or the binding-energy depth) due to the strong-coupling dynamics, are unknown, we have not been able to calculate the total rate $\Gamma_{\Pi^{0}}^{\text {total }}$ and width of the composite boson $\Pi^{0}$ that decays into the final states of the SM elementary particles including the channel of two gauge bosons or two fermions. ${ }^{7}$ Nevertheless, at the leading order (tree-level) of gauge interactions, we are able to calculate the rates $\Gamma_{\Pi^{0} \rightarrow \gamma \gamma, \gamma Z, \ldots}$ (5.29) of the composite boson $\Pi^{0}$ decaying into two gauge bosons as diboson final states,

$$
\Pi^{0} \rightarrow \gamma \gamma, \gamma Z^{0}, W^{+} W^{-}, Z^{0} Z^{0}, \cdots
$$

These diboson channels (5.35) are expected to be the most energetically favorable and largest branching ratio,

$$
B_{\Pi^{0} \rightarrow \gamma \gamma, \gamma Z^{0}, W^{+} W^{-}, Z^{0} Z^{0}}=\frac{\Gamma_{\Pi^{0} \rightarrow \gamma \gamma, \gamma Z^{0}, W^{+} W^{-}, Z^{0} Z^{0}}}{\Gamma_{\Pi^{0}}^{\mathrm{total}}} .
$$

Moreover, in high-energy experiments, the diboson final state and its kinematics might be more easily identified than the final state of two fermions (quarks or two jets) due to the background of the QCD dynamics.

\footnotetext{
${ }^{7}$ In ref. [20], we have some discussions of composite boson decaying into the final state of two quarks.
} 
The total decay rate and width of the composite boson, as well as the branching ratios of decay channels, are very important for the collider phenomenology of the composite boson. However, the following ratios of the branching ratios (5.36) for different decay channels (5.35), "relative branching ratio"

$$
\frac{\Gamma_{\Pi^{0} \rightarrow \gamma \gamma, \gamma Z^{0}, W^{+} W^{-}, Z^{0} Z^{0}}}{\Gamma_{\Pi^{0} \rightarrow \gamma \gamma}}=\frac{B_{\Pi^{0} \rightarrow \gamma \gamma, \gamma Z^{0}, W^{+} W^{-}, Z^{0} Z^{0}}}{B_{\Pi^{0} \rightarrow \gamma \gamma}}=\frac{\Gamma_{\Pi^{0} \rightarrow \gamma \gamma, \gamma Z^{0}, W^{+} W^{-}, Z^{0} Z^{0}} / \Gamma_{\Pi^{0}}^{\text {total }}}{\Gamma_{\Pi^{0} \rightarrow \gamma \gamma} / \Gamma_{\Pi^{0}}^{\text {total }}}
$$

depend only on the SM gauge couplings $g$ and $g^{\prime}$ at the energy scale $M_{\Pi}$, given by the effective Lagrangian (4.8). Following these discussions and using eqs. (5.31)-(5.34), we can approximately estimate the decay-rate ratios (5.37)

$$
\begin{aligned}
\Gamma_{\Pi^{0} \rightarrow \gamma Z^{0}} / \Gamma_{\Pi^{0} \rightarrow \gamma \gamma} & =\frac{\left(9 / 10-\sin ^{2} \theta_{W}\right)^{2}}{\sin ^{2} 2 \theta_{W}} \approx 0.63, \\
\Gamma_{\Pi^{0} \rightarrow W^{+} W^{-}} / \Gamma_{\Pi^{0} \rightarrow \gamma \gamma} & \approx\left(\frac{9}{40 \sin ^{2} \theta_{W}}\right)^{2} \approx 0.96, \\
\Gamma_{\Pi^{0} \rightarrow Z^{0} Z^{0}} / \Gamma_{\Pi^{0} \rightarrow \gamma \gamma} & =\left(\frac{(9 / 10)-(9 / 5) \sin ^{2} \theta_{W}+\sin ^{4} \theta_{W}}{\sin ^{2} 2 \theta_{W}}\right)^{2} \approx 0.58,
\end{aligned}
$$

where the value $\sin ^{2} \theta_{W} \approx 0.23$ is approximately adopted to obtain numbers. These relations provide a possibility to verify the validity of such an effective theory of composite particles, if the resonance of diphoton channel and other diboson channels are also observed and measured in high-energy experiments, for instance the on-going ATLAS and CMS experiments in the LHC $p p$ collisions.

It is also possible that the composite boson $\Pi^{0}$ decays into the dijet final state of two quarks which was already discussed in refs. [20, 21]. Suppose that the neutral composite meson $\Pi^{0}$ is produced by $p p$ collisions at the LHC, its resonance location $\mathcal{M}_{\text {all channels }}=$ $M_{\Pi^{0}}$, and the rate of each decay channel depends on the values of the mass $M_{\Pi^{0}}$, decay constant $F_{\Pi}$ and SM gauge couplings at these scales. Which channel is more relevant for detections, depending not only on its theoretical branching ratio in principle, but also on its experimental measurement in practice.

The same analysis and discussion can be generalized to the decay rates of neutral composite bosons $\Pi^{0}$ made of charged lepton and/or neutrino pair (4.5) in the lepton channel. Their decay constants and masses should be approximately at the same scale of the $\Pi^{0}$ decay constant $F_{\Pi}$ and mass $M_{\Pi^{0}}$. Their decay rates can be obtained by eqs. (5.24)(5.30) for $N_{c}=1$, couplings $Q^{i}$ and $g_{V}^{i}$ of the lepton sector in the SM Lagrangian (4.7). As an example for the two-photon final state, the rate should be $(5 / 9)^{2} N_{c}^{2}=25 / 9$ time smaller than the rate (5.31) of the quark channel. However, the ratios of branching ratios for different diboson channels are similar to eq. (5.38), but modified accordingly to the leptonic $Q^{i}$ and $g_{V}^{i}$ values. Beside, in the LHC $p p$ collision, the production probability of leptonic composite bosons is smaller than hadronic one, due to the leptonic production rate is proportional to the small fine-structure constant $\alpha$. 


\section{5 $\quad \Pi^{ \pm}$and other composite boson decays}

Equations (5.1) and (5.2) of the PCAC are not applicable for the charged composite bosons $\Pi^{ \pm}$decay. Therefore, we cannot use the analogy of the QCD charged pion $\pi^{ \pm}$decays obtain the rates of $\Pi^{ \pm}$decay to either two quarks (jets) or two leptons,

$$
\Pi^{+} \rightarrow t+b, \cdots ; \tau^{+}+\nu_{\tau}, \cdots
$$

and charge-conjugated processes. In the quark case, the composite bosons $\Pi^{ \pm}$are made of quarks (4.1), and in the lepton case the composite bosons $\Pi^{ \pm}$are made of leptons (4.5). Nevertheless, it is expected that the $\Pi^{ \pm}$-decay channel to the heaviest fermions, top quark or tau lepton, should be most favorable. Suppose that the charged composite meson $\Pi^{ \pm}$is produced by $p p$ collisions at the LHC, its resonance locates at the invariant mass $\mathcal{M}_{\mathrm{qq}} \approx M_{\Pi^{ \pm}}$of the dijet final state or other possible final states produced by two quarks (two jets). Whereas the charged lepton channel implies the final state of $\tau^{ \pm}$lepton of energy $\sim M_{\Pi^{ \pm}} / 2$ and missing energy carried away by $\nu_{\tau^{-} \text {-neutrino. }}$

In eqs. (5.6) and (5.7), the scalar bosons form composite quarkonium states $\bar{u} u$ and $\bar{d} d$ that carry the same quantum numbers of QCD quarknium states, however, have much larger masses $\sim M_{\Pi^{0}}$. It is expected that apart from different kinematic threshold, these composite quarkonium states in principle undergo all decay channels of QCD quarkonium states. Recall that the direct decays of composite bosons $\Pi^{ \pm}$and quarkonium states into dijets or dilepton without an intermediate state were preliminarily discussed [20,21]. On the basis of the SM chiral gauge symmetries and vector-like composite fermion content, there are also other possible final decay channels, analogously to those studied in the effective theory of QCD at low energies. These will be issues in future studies.

\section{Composite fermion decay and annihilation channels}

In this section 6 , we turn to discussions of decay and annihilation channels of composite fermions, see section 4.1, on the basis of the effective Lagrangian (4.8) of their kinetic terms and interactions to SM gauge bosons. We show the peculiar characteristics of composite fermions decay. It could be a possibility and possible criterion for the experimental verification or falsification of the effective theory of composite particles in the UV-domain of strong four-fermion couplings.

\subsection{Composite fermions decay}

\subsubsection{Decay into two gauge bosons and a quark}

For the sake of simplicity, we adopt the first quark family (4.1)-(4.3) to discuss the decay of composite Dirac fermions, and discussions can be generalized to other families including leptons. The composite Dirac fermions $\Psi_{D}$ (4.3) should be more massive than their constituents of composite boson $\mathcal{A}^{i}$ (4.1), i.e., $M_{F}>M_{\Pi}^{0, \pm}$. They decay into the SM elementary particles via the intermediate composite-boson states $\Pi^{0}$ (neutral decay channel) or $\Pi^{ \pm}$(charged decay channel) or quarkonium states in eqs. (5.6) and (5.7). Suppose that at the present energy of $p p$ collisions at the LHC, composite Dirac fermions produced are 
non-relativistic particles, and we consider them to be approximately at rest in the CM frame of $p p$ collision. The neutral decay channels are, see figure 5 , for the $u$-quark channel

$$
\Psi_{D} \rightarrow \Pi^{0}+\left[\bar{u}_{L}, u_{R}\right] \rightarrow\left(\gamma \gamma, \gamma Z^{0}, W^{+} W^{-}, Z^{0} Z^{0}\right)+\text { a jet }
$$

and the $d$-quark channel given by $u \rightarrow d$. This indicates that a composite Dirac fermion $\Psi_{D}$ decays into a composite meson $\Pi^{0}$ and a fundamental Dirac fermion, $u$-quark $\left[\bar{u}_{L}, u_{R}\right]$ or $d$-quark $\left[\bar{d}_{L}, d_{R}\right]$. The latter is an ultra-relativistic quark, $E_{f} \approx\left|\mathbf{p}_{f}\right|$, ending as a jet in the final states. And the former is a composite meson, $E_{\Pi^{0}}=\left(\mathbf{p}_{\Pi^{0}}^{2}+M_{\Pi^{0}}^{2}\right)^{1 / 2}$, appearing as an intermediate and metastable state for a short time $\sim 1 / M_{\Pi^{0}}$ then decays into two photons of the energy $E_{\gamma}=\left|\mathbf{p}_{\gamma}\right|$ or other diboson channels (5.35) of two SM gauge bosons $G$ and $G^{\prime}$. In the CM frame of the $p p$ collision, the composite fermion is approximately at rest, and decays into a quark (jet) and a composite boson moving apart in opposite directions $\left(\mathbf{p}_{f}=-\mathbf{p}_{\Pi^{0}}\right)$. The kinematic distribution of final states is described by the invariant mass $\mathcal{M}_{\text {inv }} \approx M_{F}$,

$$
\begin{aligned}
M_{F} & =E_{f}+E_{\Pi^{0}} \approx\left|\mathbf{p}_{\Pi^{0}}\right|+\left(\mathbf{p}_{\Pi^{0}}^{2}+M_{\Pi^{0}}^{2}\right)^{1 / 2} \\
\mathbf{p}_{f} & =-\mathbf{p}_{\Pi^{0}}=-\left(\mathbf{p}_{\gamma_{1}}+\mathbf{p}_{\gamma_{2}}\right) \\
\left|\mathbf{p}_{\Pi^{0}}\right| & \approx\left[\left|\mathbf{p}_{\gamma_{1}}\right|^{2}+\left|\mathbf{p}_{\gamma_{1}}\right|^{2}+2\left|\mathbf{p}_{\gamma_{1}}\right|\left|\mathbf{p}_{\gamma_{2}}\right| \cos \theta_{\gamma_{1} \gamma_{2}}\right]^{1 / 2}
\end{aligned}
$$

where $\theta_{\gamma_{1} \gamma_{2}}$ is the angle between the momenta $\mathbf{p}_{\gamma_{1}}$ and $\mathbf{p}_{\gamma_{2}}$ of two photons or other diboson states (6.1). The rate $\Gamma_{\Psi_{D} \rightarrow G G^{\prime}+\text { jet }}$ of composite fermion decay (6.1) should be the product of $\Pi_{0^{-}}$decay rate $\Gamma_{\Pi_{\Pi^{0}} \rightarrow G G^{\prime}}(5.31)-(5.34)$ and the rate $\Gamma_{\Psi_{D} \rightarrow \Pi^{0}+\text { a quark }}$ of $\Psi_{D}$ decaying into a quark and $\Pi_{0}$, namely

$$
\Gamma_{\Psi_{D} \rightarrow G G^{\prime}+\mathrm{a} \text { quark }} \propto \Gamma_{\Psi_{D} \rightarrow \Pi^{0}+\mathrm{a} \text { quark }} \times \Gamma_{\Pi_{\Pi^{0}} \rightarrow G G^{\prime}} .
$$

This implies the ratios:

$$
\begin{aligned}
\Gamma_{\Psi_{D} \rightarrow \gamma Z^{0}+\mathrm{a} \text { quark }} / \Gamma_{\Psi_{D} \rightarrow \gamma \gamma+\mathrm{a} \text { quark }} & \propto \Gamma_{\Pi^{0} \rightarrow \gamma Z^{0}} / \Gamma_{\Pi^{0} \rightarrow \gamma \gamma}, \\
\Gamma_{\Psi_{D} \rightarrow W^{+} W^{-}+\mathrm{a} \text { quark }} / \Gamma_{\Psi_{D} \rightarrow \gamma \gamma+\mathrm{a} \text { quark }} & \propto \Gamma_{\Pi^{0} \rightarrow W^{+} W^{-}} / \Gamma_{\Pi^{0} \rightarrow \gamma \gamma}, \\
\Gamma_{\Psi_{D} \rightarrow Z^{0} Z^{0}+\mathrm{a} \text { quark }} / \Gamma_{\Psi_{D} \rightarrow \gamma \gamma+\mathrm{a} \text { quark }} & \propto \Gamma_{\Pi^{0} \rightarrow Z^{0} Z^{0}} / \Gamma_{\Pi^{0} \rightarrow \gamma \gamma} .
\end{aligned}
$$

are similar to the ratios (5.38). If the bosonic resonance (5.35) is observed and its invariant mass is determined, the resonance of composite fermion decay (6.1) could possibly be identified by measuring such a peculiar final-state kinematics (6.2) of a single jet and two photons or other diboson states $G$ and $G^{\prime}$.

\subsubsection{Decay into two gauge bosons and a lepton}

Due to the four-fermion interaction of quark-lepton sector, see eq. (2.18) and section 4.1.3, the composite fermions (4.6) composed by a hadronic composite boson and a lepton are also formed in the UV-domain. We generalize the analysis and discussion in the previous section 6.1.1 to the decay of composite fermion (4.6) composed by a hadronic composite 


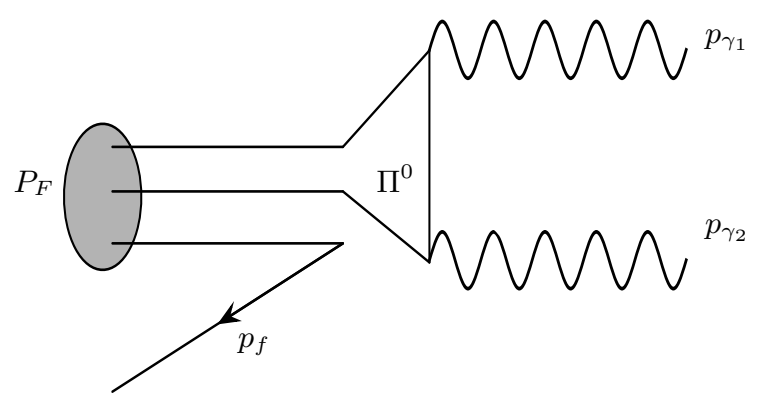

Figure 5. We show the diagram of a composite fermion (three parallel solid lines) decaying to an SM elementary quark or lepton (the solid line with an arrow) and a composite boson, the latter decays into two SM gauge bosons (wave lines), see figure 4 . The composite boson $\Pi^{0}$ is an intermediate state. $p_{f}$ represents the energy momentum of quark or lepton. $p_{\gamma_{1}}$ and $p_{\gamma_{2}}$ represent the energy momentum of two photons or two gauge bosons $G$ and $G^{\prime}$. The composite fermion is heavier than composite boson $\Pi^{0}, M_{F}>M_{\Pi^{0}}$, and the energy-momentum of composite fermion $E_{F}=M_{F}$ at its rest frame $\left(\mathbf{P}_{F}=0\right)$, as described in eq. (6.2).

boson $\Pi^{0}$ and a charged lepton (or a neutrino). The decay process is also represented by figure 5. For the e-lepton channel,

$$
\Psi_{D} \rightarrow \Pi^{0}+\left[\bar{e}_{L}, e_{R}\right] \rightarrow\left(\gamma \gamma, \gamma Z^{0}, W^{+} W^{-}, Z^{0} Z^{0}\right)+\text { an electron }
$$

and the $\nu_{e}$-lepton channel is given by $e \rightarrow \nu$. This indicates that a composite Dirac fermion $\Psi_{D}$ (4.6) decays into a composite meson $\Pi^{0}$ and a fundamental Dirac fermion, a charged lepton $\ell=\left[\bar{\ell}_{L}, \ell_{R}\right]$ or a neutrino $\nu_{\ell}$. The latter is an ultra-relativistic lepton. The kinematic threshold and distribution are the same as the one (6.1) in pure hadronic channel (6.2). However, the neutrino in the final states carries away mixing energy and momentum. The

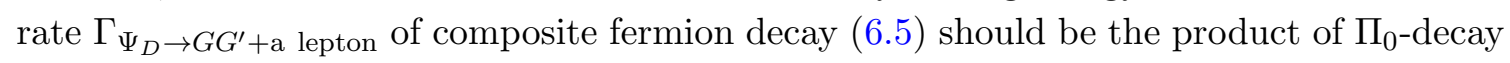
rate $\Gamma_{\Pi_{\Pi^{0}} \rightarrow G G^{\prime}}$ (5.31)-(5.34) and the rate $\Gamma_{\Psi_{D} \rightarrow \Pi^{0}+\text { a lepton }}$ of $\Psi_{D}$ decaying into a lepton and $\Pi_{0}$, namely

$$
\Gamma_{\Psi_{D} \rightarrow G G^{\prime}+\text { a lepton }} \propto \quad \Gamma_{\Psi_{D} \rightarrow \Pi^{0}+\text { a lepton }} \times \Gamma_{\Pi_{\Pi^{0}} \rightarrow G G^{\prime}}
$$

This implies the ratios:

$$
\begin{aligned}
\Gamma_{\Psi_{D} \rightarrow \gamma Z^{0}+\mathrm{a} \text { lepton }} / \Gamma_{\Psi_{D} \rightarrow \gamma \gamma+\mathrm{a} \text { lepton }} & \propto \Gamma_{\Pi^{0} \rightarrow \gamma Z^{0}} / \Gamma_{\Pi^{0} \rightarrow \gamma \gamma}, \\
\Gamma_{\Psi_{D} \rightarrow W^{+} W^{-}+\mathrm{a} \text { lepton }} / \Gamma_{\Psi_{D} \rightarrow \gamma \gamma+\text { a lepton }} & \propto \Gamma_{\Pi^{0} \rightarrow W^{+} W^{-}} / \Gamma_{\Pi^{0} \rightarrow \gamma \gamma}, \\
\Gamma_{\Psi_{D} \rightarrow Z^{0} Z^{0}+\text { a lepton }} / \Gamma_{\Psi_{D} \rightarrow \gamma \gamma+\text { a lepton }} & \propto \Gamma_{\Pi^{0} \rightarrow Z^{0} Z^{0}} / \Gamma_{\Pi^{0} \rightarrow \gamma \gamma}
\end{aligned}
$$

are similar to the ratios (5.38).

The decay processes (6.1) and (6.5) discussed in this section show the peculiar characteristics of the effective theory of composite bosons and fermions in the UV-domain of strong four-fermion couplings. These processes are relevant to and can be checked by the on-going high-energy experiments in the LHC $p p$ collision. 


\subsubsection{Other channels of composite fermion decay}

In addition, the decay channels of composite fermions via the intermediate meson state of charged composite bosons $\Pi^{ \pm}$(5.39) read

$$
\Psi_{D} \rightarrow \Pi^{+}+\left[\bar{u}_{L}, d_{R}\right]
$$

and its charge conjugate. The Weyl fields $\left[\bar{u}_{L}, d_{R}\right]$ do not form a Dirac fermion of $u$ - or $d$-quark, but pick up $u_{R}$ and $\bar{d}_{L}$ quarks from the vacuum, and end as $u$ and $d$-quark (jets) in final states. On the other hand, the charged composite mesons $\Pi^{ \pm}$, see eqs. (5.6) and (5.7), most probably decay into two quarks (5.39). Therefore, the most probable final state of composite fermion (6.8) decay is expected to be four jets formed by four quarks, which was already discussed in refs. [20,21].

These discussions can be generalized to the decay channels whose intermediate state is a composite quarkonium state $S^{0 \pm}$, instead of a composite meson state $\Pi^{0 \pm}$, see eqs. (5.6) and (5.7). Also, these discussion can be generalized to the second and third families of composite fermions.

To end this section, we mention other possible channels of composite fermion decay. Analogously to composite-fermion decay channel (6.8), the composite fermions (4.6) formed the quark-lepton interaction decay via the intermediate meson state $\Pi^{ \pm}(5.39)$

$$
\Psi_{D} \rightarrow \Pi^{+}+\left[\bar{\nu}_{L}, \ell_{R}\right]
$$

and its charge conjugate. The final states are a lepton pair and a jet pair. As for the decay of leptonic composite fermions (4.5), final states are: (i) two gauge bosons and a lepton for the neutral channel via the intermediate $\Pi^{0}$ state; (ii) four leptons for the charged channel via the intermediate $\Pi^{ \pm}$state. However these decay rates are much smaller due to the "missing" of the factor $N_{c}^{2}$ and the smallness of the fine-structure constant $\alpha$ relating to the production of leptons in the LHC $p p$ collision.

\subsection{Annihilation of two composite fermions}

We turn to qualitative discussions of two composite fermions annihilation into two SM gauge bosons $G$ and $G^{\prime}$, which should be less measurable process compared with a composite fermion decay, in the experimental view of producing two massive composite fermions and detecting final states. Analogously to the annihilation of electron and positron into two photons in the QED, a composite Dirac fermion and its antiparticle annihilates to two photons, and other massive gauge bosons, see figure 6 ,

$$
\Psi_{D}+\Psi_{D}^{c} \rightarrow \gamma \gamma, \gamma Z^{0}, W^{+} W^{-}, Z^{0} Z^{0}, \cdots
$$

where the composite Dirac fermion $\Psi_{D}$ has an electric charge $Q=(2 / 3,-1 / 3,-1)$ and mass $M_{F}$. Apart from the diphoton final state, the final states of energetic dibosons $W^{+} W^{-}$ and $Z^{0} Z^{0}$ are two "fat" jets in opposite directions, each of them is made by two energetic quarks, or these two bosons can decay into the final states of two lepton pairs. While in the channel $\Psi_{D}+\Psi_{D}^{c} \rightarrow \gamma Z^{0}$, the final states are an energetic photon and a "fat" jet or an energetic photon and a lepton pair. 

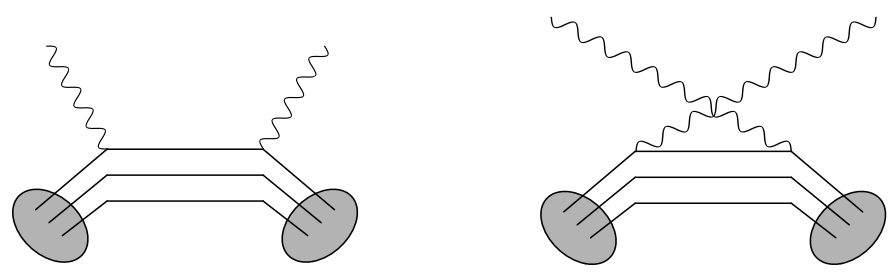

Figure 6. These diagrams show the annihilation of two composite fermions (three solid lines) into two SM gauge bosons (two wave lines).

Given by the mass-energy of two annihilating composite Dirac fermions, the kinematic mass-energy of final states must be larger than $2 M_{F}$. This is not an invariant energymass representing the resonance of an unstable composite particle. Suppose that massive composite Dirac fermions produced by the LHC $p p$-collision at present energies are nonrelativistic particles,

$$
E_{F}=\left(\left|\mathbf{p}_{F}\right|^{2}+M_{F}^{2}\right)^{1 / 2} \approx M_{F}+\left|\mathbf{p}_{F}\right|^{2} /\left(2 M_{F}\right),
$$

assuming the mass $M_{F}$ is large enough. Two SM gauge bosons $G$ and $G^{\prime}$ in the final state (6.10) are ultra-relativistic, the cross-section $\sigma_{D}$ of annihilating channel (6.10) can be approximately estimated by using the Dirac rate of point-like electron and positron annihilating to two photons with the replacement $m_{e} \rightarrow M_{F}$ and $\alpha \rightarrow Q^{2} \alpha$,

$$
\sigma_{D} \sim \pi r_{0}^{2} / v, \quad r_{0}=Q^{2} \alpha / M_{F}, \quad v=\left|\mathbf{p}_{F}\right| / M_{F} \ll 1,
$$

up to the form-factors $Z_{L, R}^{S}$ (4.2) of composite Dirac fermions. The annihilation rate $\Gamma_{D}$ per unit time

$$
\Gamma_{\Psi_{D} \Psi_{D}^{c} \rightarrow \gamma \gamma} \approx \sigma_{D} v n \sim 2 \pi r_{0}^{2} n \sim\left(Q^{2} \alpha\right)^{2} M_{F}
$$

where the number density of the produced composite fermions $n \sim M_{F}^{3} / \pi$ is assumed. Analogously to the positronium state of electron and positron pair in the QED, it is possible that the composite fermion and its antiparticle form an intermediate unstable Coulomb bound state, then decaying into photons or other massive gauge bosons. The spin singlet of the bound state decays into two photons (even number of photons) with the probability $\sim\left(Q^{2} \alpha\right)^{5} M_{F}$, The spin triplet of the bound state decays into three photons (odd number of photons) with the probability $\sim\left(Q^{2} \alpha\right)^{6} M_{F}$. In these discussions, we adopt two-photon final state. As for other channels of two SM gauge bosons $G$ and $G^{\prime}$ (6.10), in terms of their SM gauge couplings in the effective Lagrangian (4.8), we can obtain the ratios of different annihilation processes:

$$
\Gamma_{\Psi_{D} \Psi_{D}^{c} \rightarrow \gamma Z^{0}} / \Gamma_{\Psi_{D} \Psi_{D}^{c} \rightarrow \gamma \gamma}, \quad \Gamma_{\Psi_{D} \Psi_{D}^{c} \rightarrow W^{+} W^{-}} / \Gamma_{\Psi_{D} \Psi_{D}^{c} \rightarrow \gamma \gamma}, \quad \Gamma_{\Psi_{D} \Psi_{D}^{c} \rightarrow Z^{0} Z^{0}} / \Gamma_{\Psi_{D} \Psi_{D}^{c} \rightarrow \gamma \gamma}
$$

depending only on the SM gauge couplings $g$ and $g^{\prime}$ (4.8) at the mass scale $M_{F}$, similarly to the ratios (5.38).

The following annihilation channels of two composite fermions with different charges or zero charge are also possible,

$$
\begin{aligned}
\Psi_{D}^{-1 / 3}+\Psi_{D}^{c 2 / 3} & \rightarrow W^{-} \gamma, W^{-} Z^{0}, \cdots \\
\Psi_{D}^{0}+\Psi_{D}^{0} & \rightarrow W^{+} W^{-}, Z^{0} Z^{0}, \cdots
\end{aligned}
$$


and their charged conjugates. In addition, it is known that the annihilation of electron and positron, through an intermediate $\gamma$-photon or $Z^{0}$-boson, produce a pair of particle and antiparticle in the SM. Analogously, a composite fermion and its antiparticle annihilates, through an intermediate photon or $Z^{0}$-boson, and produce a pair of SM elementary particle and its antiparticle,

$$
\Psi_{D}+\Psi_{D}^{c} \rightarrow f+f^{c},
$$

where the final state is an energetic lepton pair or quark pair, the former is the dilepton channel and the latter is the dijet channel. Similarly to the channels (6.15) two composite Dirac fermions with different charges or zero charge can annihilate, through an intermediate charged boson $W^{ \pm}$or neutral boson $Z^{0}$, to the pair of two SM elementary fermions.

The analogous discussions on these possible processes can be also made for the case of the composite fermion $\Psi_{S}$ (singlet) coupling to the $\gamma$ and $Z^{0}$, see eq. (4.8); as well as for the case of composite bosons $\mathcal{A}^{i}$ coupling to the $\gamma, Z^{0}$ and $W^{ \pm}$, see eq. (4.9). In the effective Lagrangian (4.8) and (4.9), we neglect the interactions between the composite particles, e.g., the composite fermion and boson interaction of the Yukawa type. Thus we skip the discussions on two composite Dirac fermions annihilate to two composite bosons $\mathcal{A}$ and $\mathcal{A}^{\dagger}$. Limited by the lengthy of this article, it is impossible to discuss even qualitatively all possible processes of the effective Lagrangian (4.8) and (4.9), thus we are led to some considerations and speculations possibly relevant to experiments and /or observations.

\section{Some speculative considerations for experiments}

In this article, for readers' convenience, we first give a very brief review of effective fourfermion operators and effective theories of elementary and composite particles in IR and UV scaling domains. We then focus the discussions on the decay and annihilation channels of composite bosons and fermions into the final states of the SM gauge bosons, leptons and quarks, in connection with the searches of high-energy experiments, like the ATLAS and CMS. The possibilities and possible criteria are provided for the verification or falsification of such an effective theory of massive composite particles and SM gauge couplings in the UV-domain of strong four-fermion couplings. Obviously, in addition to those processes discussed in this article, there are other possible experimentally relevant processes of effective four-fermion operators (2.6), we end this lengthy article by making some speculative considerations for experiments.

\subsection{Some speculative considerations for LHC experiments}

In the LHC $p p$ collisions, the most probably channels of producing composite particles are the composite bosons and fermions (4.1)-(4.4) in the first family via the four-fermion operators $[20,21]$

$$
G\left[\left(\bar{\psi}_{L}^{i a} u_{R a}\right)\left(\bar{u}_{R}^{b} \psi_{L b}^{i}\right)+\left(\bar{\psi}_{L}^{i a} d_{R a}\right)\left(\bar{d}_{R}^{b} \psi_{L b}^{i}\right)\right]+G\left(\bar{\psi}_{L}^{i a} \nu_{R}\right)\left(\bar{\nu}_{R} \psi_{L}^{i a}\right),
$$

and the channels of producing composite particles by other quark and lepton families have smaller rate because of involving small SM gauge interactions. The last term of eq. (7.1) 
represents the interaction of quarks and dark-matter particle $\nu_{R}$, which stands for the right-handed sterile neutrino of the first fermion family.

These composite bosons and fermions could be experimentally verified by possibly observing their resonances and measuring their invariant masses $\left(\mathcal{M}_{\text {inv }}\right)$ and kinematic distributions of relevant final states. If the CM energy $\sqrt{s}$ of LHC pp-collisions is close to the masses $M_{\Pi^{0}}$ and $M_{F}$ of composite boson and fermion $\left(\sqrt{s} \gtrsim M_{\Pi^{0}}, M_{F}\right)$, then the invariant mass $\mathcal{M}_{\text {inv }} \sim M_{\Pi^{0}}$ or $\mathcal{M}_{\text {inv }} \sim M_{F}$. The mass scales $M_{\Pi^{0}}$ and $M_{F}$ can only be determined by high-energy experiments, though we have the theoretical relation $M_{F}>M_{\Pi} \propto \mathcal{E}_{\xi}$ of eq. (4.12) and preliminary theoretical estimation (3.19) on the characteristic energy scale $\mathcal{E}_{\xi} \gtrsim 5 \mathrm{TeV}$ of the UV-domain.

Suppose that the recent ATLAS and CMS preliminary results [69, 70] of diboson resonances (dijets tagged by two bosons) with invariant masses in the energy range from 1.3 to $3.0 \mathrm{TeV}$ could be further confirmed. These resonances are expected to be also seen in the channels of four quark jets, whose invariant mass and event rate should be larger, provided that these resonances are attributed to massive composite Dirac fermions at this energy range. The CMS result [71] of resonances with final states being two jets could include the event of four quark jets, two of them are geometrically close together to form a "wide jet", which should be tagged through a study of its substructure and flavor. Moreover, if composite Dirac fermions are formed by the last operator in eq. (7.1), in addition to jets in final state, dark-matter particles $\nu_{R}$ carry away missing energy-momentum [72, 73]. Similar discussions are applied for the case of composite bosons.

Due to the $W^{ \pm}$- and $Z^{0}$-boson couplings $g_{2}$ to two constituent quarks $(u, d)$ of composite fermions, in particular $W^{ \pm}$-boson coupling to $S U_{L}(2)$ doublet $\psi_{L}^{i a}=\left(u_{L}^{a}, d_{L}^{a}\right)$, massive composite Dirac fermions have the following decay channels of final states: (i) dijets tagged by two highly boosted bosons WW, WZ or ZZ produced by high-energy constituent quarks $(u, d)$ of composite fermions, together with additional quark jets; (ii) four quark jets formed by four high-energy constituent quarks $(u, d)$ of a composite fermion with a peculiar kinematic distribution $[20,21]$. It is expected that the former should have smaller rate because of the SM gauge coupling $g_{2}$, although we have not yet been able to calculate the rates of these channels. In these two aforementioned channels (i) and (ii), the final states can also be high-energy leptons, however the branching ratio of $W^{ \pm}$and $Z^{0}$ decaying into leptons is about several times smaller than that to hadrons (jets) [67]. The composite fermion can also decay in the channel of $W$ and Higgs (WH) bosons [68], where the Higgs boson is produced by $u, d$-quarks fusing into a top-quark pair via a gluon, and its production rate is then related to the QCD coupling $\alpha_{s}=g_{3}^{2} / 4 \pi$. Similar discussions are applied for the case of composite bosons.

\subsection{Sterile neutrinos interacting with SM particles at high energies}

Last but not least, all sterile neutrinos $\left(\nu_{R}^{i}, \nu_{R}^{i c}\right)$ and SM gauge-singlet (neutral) states of massive composite fermions, e.g., $\mathbf{\Psi}_{D} \sim\left[\bar{\nu}_{R}^{\ell},\left(\bar{\ell}_{L}^{i} \nu_{R}^{\ell}\right) \ell_{L i}\right]$, can be possible candidates of warm and cold dark matter $[19,21]$. They can couple or decay into the SM elementary particles in the following ways. (i) SM gauge-singlet (neutral) states of composite Dirac fermions become unstable and decay into SM elementary particles. (ii) Sterile neutrinos interact 
with SM elementary particles via the last term of eq. (7.1) for $\psi_{L}^{i}$ being quark or lepton $S U_{L}(2)$-doublets. (iii) The terms in eqs. (2.16) and (2.17) give the interactions

$$
G\left[\left(\bar{\ell}_{L}^{i} \nu_{R}^{\ell}\right)\left(\bar{\nu}_{R}^{\ell} \ell_{L i}\right)+\left(\bar{\nu}_{R}^{\ell c} \ell_{R}\right)\left(\bar{\ell}_{R} \nu_{R}^{\ell c}\right)+\left(\bar{\nu}_{R}^{\ell c} u_{a, R}^{\ell}\right)\left(\bar{u}_{a, R}^{\ell} \nu_{R}^{\ell c}\right)+\left(\bar{\nu}_{R}^{\ell c} d_{a, R}^{\ell}\right)\left(\bar{d}_{a, R}^{\ell} \nu_{R}^{\ell c}\right)\right],
$$

among sterile neutrinos $\nu_{R}^{\ell}, \nu_{R}^{\ell c}$ (dark matter) and SM elementary particles, where the lepton $S U_{L}(2)$ doublets $\ell_{L}^{i}=\left(\nu_{L}^{\ell}, \ell_{L}\right)$, singlets $\ell_{R}$ and the conjugate fields of sterile neutrinos $\nu_{R}^{\ell c}=i \gamma_{2}\left(\nu_{R}^{\ell}\right)^{*}(\ell=e, \mu, \tau)$, and quark fields $u_{a, R}^{\ell}=(u, c, t)_{a, R}$ and $d_{a, R}^{\ell}=(d, s, b)_{a, R}$.

The four-fermion coupling $G$ in eqs. (7.1) and (7.2) is unique. Therefore, it is expected that at the same energy scale $M_{F}>M_{\Pi} \propto \mathcal{E}_{\xi}$ (4.12), at which composite boson and fermion (4.1)-(4.4) appear as resonances in the LHC $p p$ collisions, leptonic composite boson $\left(\bar{e}_{R} \nu_{R}^{e c}\right)$ or $\left(\bar{\nu}_{R}^{e} e_{L}\right)$ and composite fermion $\left[\bar{\nu}_{R}^{e c},\left(\bar{e}_{R} \nu_{R}^{e c}\right) e_{R}\right]$ or $\left[\bar{e}_{L},\left(\bar{\nu}_{R}^{e} e_{L}\right) \nu_{R}^{e}\right]$ should be formed by high-energy sterile neutrino inelastic collisions, e.g. $\nu_{R}^{e}+\bar{\nu}_{R}^{e} \rightarrow e^{-}+e^{+}$via the first or second interaction in eq. (7.2). Then these leptonic composite particles decay and produce electrons and positrons. This may account for an excess of cosmic ray electrons and positrons around $\mathrm{TeV}$ scale [74-77] in space laboratories. In addition, recent AMS-02 results [78] show that at $\mathrm{TeV}$ scale the energy-dependent proton flux changes its power-law index. This implies that there would be "excess" $\mathrm{TeV}$ protons whose origin could be also explained by the resonance of composite bosons and fermions due to the interactions (7.1) and (7.2) of dark-matter and normal-matter particles.

We also expect that at the same energy scale $M_{F}>M_{\Pi} \propto \mathcal{E}_{\xi}$ (4.12), the four-fermion interactions of the last term in eq. (7.1), the third and fourth terms in eq. (7.2) form composite fermions made by $u, d$-quarks and sterile neutrino $\nu_{R}$, e.g., $\left[\bar{\psi}_{L a}^{i},\left(\bar{\nu}_{R} \psi_{L a}^{i}\right) \nu_{R}\right]$, $\left[\bar{\nu}_{R}^{\ell c},\left(\bar{u}_{a R} \nu_{R}^{\ell c}\right) u_{a R}\right]$ or $\left[\bar{u}_{a L},\left(\bar{\nu}_{R}^{\ell} u_{a L}\right) \nu_{R}^{\ell}\right]$. These composite states should appear as resonances by high-energy sterile neutrinos inelastic collisions with nucleons (xenon) at the largest cross-section, then resonances decay and produce some other detectable SM particles in underground laboratories [79]. Similarly, in the ICECUBE experiment [80], we expect the events that neutrinos change (lose) their directions (energies) by the first term of the interaction (2.16) to form the resonances of composite bosons and fermions at the same energy scale $M_{F}>M_{\Pi} \propto \mathcal{E}_{\xi}$ (4.12). In these inelastic collisions, if the accessible CM energy $\sqrt{s}>M_{\Pi, F}$, the cross section for the allowed inelastic processes forming massive composite bosons and fermions will be geometrical in magnitude, of order $\sigma_{\text {com }} \sim 4 \pi / M_{\Pi, F}^{2}$ in the CM frame where massive composite bosons and fermions are approximately at rest.

\subsection{Further studies}

By contrast with the low-energy effective theory of SM elementary particles in the IRdomain of weak four-fermion coupling, we have to confess that the present analysis of the effective theory of composite particles in the UV-domain of strong four-fermion coupling are completely preliminary, due to its non-perturbative nature from the theoretical point view. Adequate non-perturbative methods both analytical approaches and numerical algorithms are necessary. This is analogous to the theoretical aspects of low-energy hadron physics of non-perturbative QCD. On the other hand, from experimental point of view, it is nontrivial to proceed physically sensible final-state selection and relevant data analysis in high-energy 
experiments, in addition to searching for high-energy collisions and accumulating enough data for significant analysis. However, without high-energy experiments, the characteristic energy scale $M_{F}>M_{\Pi} \propto \mathcal{E}_{\xi}$ (4.12) of effective composite-particle theory cannot even be determined at all. It is worthwhile to mention that similar to the analogy between the Higgs mechanism and Bardeen-Cooper-Schrieffer (BCS) superconductivity, we are studying an analogy between the effective theory discussed in this article and the BCS-BEC (BoseEinstein condensate) crossover and unitary Fermi gas of strong-interacting electrons [81], which is expected to observe in optical lattice.

\section{Acknowledgments}

The author thanks Prof. Zhiqing Zhang for discussions on the LHC physics, and Prof. Hagen Kleinert for discussions on the scaling domains of the IR- and UV-stable fixed points of quantum field theories.

Open Access. This article is distributed under the terms of the Creative Commons Attribution License (CC-BY 4.0), which permits any use, distribution and reproduction in any medium, provided the original author(s) and source are credited.

\section{References}

[1] Y. Nambu and G. Jona-Lasinio, Dynamical Model of Elementary Particles Based on an Analogy with Superconductivity. 1., Phys. Rev. 122 (1961) 345 [INSPIRE].

[2] F. Englert and R. Brout, Broken Symmetry and the Mass of Gauge Vector Mesons, Phys. Rev. Lett. 13 (1964) 321 [INSPIRE].

[3] P.W. Higgs, Broken symmetries, massless particles and gauge fields, Phys. Lett. 12 (1964) 132 [INSPIRE].

[4] P.W. Higgs, Broken Symmetries and the Masses of Gauge Bosons, Phys. Rev. Lett. 13 (1964) 508 [InSPIRE].

[5] P.W. Higgs, Spontaneous Symmetry Breakdown without Massless Bosons, Phys. Rev. 145 (1966) 1156 [INSPIRE].

[6] G.S. Guralnik, C.R. Hagen and T.W.B. Kibble, Global Conservation Laws and Massless Particles, Phys. Rev. Lett. 13 (1964) 585 [inSPIRE].

[7] T.W.B. Kibble, Symmetry breaking in nonAbelian gauge theories, Phys. Rev. 155 (1967) 1554 [INSPIRE].

[8] ATLAS collaboration, Observation of a new particle in the search for the Standard Model Higgs boson with the ATLAS detector at the LHC, Phys. Lett. B 716 (2012) 1 [arXiv: 1207.7214] [INSPIRE].

[9] CMS collaboration, Observation of a new boson at a mass of $125 \mathrm{GeV}$ with the CMS experiment at the LHC, Phys. Lett. B 716 (2012) 30 [arXiv:1207.7235] [INSPIRE].

[10] ATLAS collaboration, Search for resonances in diphoton events at $\sqrt{s}=13 \mathrm{TeV}$ with the ATLAS detector, JHEP 09 (2016) 001 [arXiv:1606.03833] [INSPIRE]. 
[11] CMS collaboration, Search for Resonant Production of High-Mass Photon Pairs in Proton-Proton Collisions at $\sqrt{s}=8$ and 13 TeV, Phys. Rev. Lett. 117 (2016) 051802 [arXiv: 1606. 04093] [INSPIRE].

[12] S.-S. Xue, Vectorlike $W^{ \pm}$-boson coupling at TeV and third family fermion masses, Phys. Rev. D 93 (2016) 073001 [arXiv:1506.05994] [INSPIRE].

[13] S.-S. Xue, Hierarchy spectrum of SM fermions: from top quark to electron neutrino, JHEP 11 (2016) 072 [arXiv:1605.01266] [INSPIRE].

[14] S.-S. Xue, A lattice chiral theory with multi-fermion couplings, Phys. Lett. B 381 (1996) 277 [hep-lat/9605003] [INSPIRE].

[15] S.-S. Xue, A possible scaling region of chiral fermions on a lattice, Nucl. Phys. B 486 (1997) 282 [hep-lat/9605005] [INSPIRE].

[16] S.-S. Xue, Chiral gauged fermions on a lattice, Nucl. Phys. B 580 (2000) 365 [hep-lat/0002026] [INSPIRE].

[17] S.-S. Xue, A further study of the possible scaling region of lattice chiral fermions, Phys. Rev. D 61 (2000) 054502 [hep-lat/9910013] [INSPIRE].

[18] S.-S. Xue, The (11112) model on a (H1)-dimensional lattice, Phys. Rev. D 64 (2001) 094504 [hep-lat/0109002] [INSPIRE].

[19] S.-S. Xue, On the standard model and parity conservation, J. Phys. G 29 (2003) 2381 [INSPIRE].

[20] S.-S. Xue, Ultraviolet fixed point and massive composite particles in $\mathrm{TeV}$ scales, Phys. Lett. B 737 (2014) 172 [arXiv:1405.1867] [INSPIRE].

[21] S.-S. Xue, Resonant and nonresonant new phenomena of four-fermion operators for experimental searches, Phys. Lett. B 744 (2015) 88 [arXiv:1501.06844] [INSPIRE].

[22] C.T. Hill, Topcolor: Top quark condensation in a gauge extension of the standard model, Phys. Lett. B 266 (1991) 419 [INSPIRE].

[23] C.T. Hill, Topcolor assisted technicolor, Phys. Lett. B 345 (1995) 483 [hep-ph/9411426] [INSPIRE].

[24] C.T. Hill, 'Super'-Dilatation Symmetry of the Top-Higgs System, Phys. Rev. D 87 (2013) 065002 [arXiv:1211.2773] [INSPIRE].

[25] C.T. Hill, Quark and Lepton Masses from Renormalization Group Fixed Points, Phys. Rev. D 24 (1981) 691 [inSPIRE].

[26] C.T. Hill, C.N. Leung and S. Rao, Renormalization Group Fixed Points and the Higgs Boson Spectrum, Nucl. Phys. B 262 (1985) 517 [InSPIRE].

[27] J. Bagger, S. Dimopoulos and E. Masso, Renormalization Group Constraints in Supersymmetric Theories, Phys. Rev. Lett. 55 (1985) 920 [INSPIRE].

[28] W.A. Bardeen, C.T. Hill and M. Lindner, Minimal Dynamical Symmetry Breaking of the Standard Model, Phys. Rev. D 41 (1990) 1647 [INSPIRE].

[29] Y. Nambu, Model Building Based on Bootstrap Symmetry Breaking, in Proceedings of the 1989 workshop on Dynamical Symmetry Breaking, in proceedings of the 1989 Workshop on Dynamical Symmetry Breaking, T. Muta and K. Yamawaki eds., Nagoya University, Nagoya, Japan (1990). 
[30] V.A. Miransky, M. Tanabashi and K. Yamawaki, Is the $t$ Quark Responsible for the Mass of $W$ and $Z$ Bosons?, Mod. Phys. Lett. A 4 (1989) 1043 [INSPIRE].

[31] V.A. Miransky, M. Tanabashi and K. Yamawaki, Dynamical electroweak symmetry breaking with large anomalous dimension and t quark condensate, Phys. Lett. B 221 (1989) 117.

[32] H. Kleinert, Particles an Quantum Field, Chapter 26, World Scientific Publishing Company, (2016), http://klnrt.de/b6.

[33] W.J. Marciano, Heavy top quark mass predictions, Phys. Rev. Lett. 62 (1989) 2793 [INSPIRE].

[34] G. Cvetič, Top quark condensation, Rev. Mod. Phys. 71 (1999) 513 [hep-ph/9702381] [INSPIRE].

[35] C.T. Hill and E.H. Simmons, Strong dynamics and electroweak symmetry breaking, Phys. Rept. 381 (2003) 235 [Erratum ibid. 390 (2004) 553] [hep-ph/0203079] [InSPIRE].

[36] S.-S. Xue, Quantum Regge calculus of Einstein-Cartan theory, Phys. Lett. B 682 (2009) 300 [arXiv: 0902.3407] [INSPIRE].

[37] S.-S. Xue, Detailed discussions and calculations of quantum Regge calculus of Einstein-Cartan theory, Phys. Rev. D 82 (2010) 064039 [arXiv:0912.2435] [INSPIRE].

[38] S.-S. Xue, The phase structure of Einstein-Cardan theory, Phys. Lett. B 665 (2008) 54 [arXiv: 0804.4619] [INSPIRE].

[39] S.-S. Xue, The Phase and Critical Point of Quantum Einstein-Cartan Gravity, Phys. Lett. B 711 (2012) 404 [arXiv:1112.1323] [INSPIRE].

[40] H.B. Nielsen and M. Ninomiya, Absence of Neutrinos on a Lattice. 1. Proof by Homotopy Theory, Nucl. Phys. B 185 (1981) 20 [Erratum ibid. B 195 (1982) 541] [InSPIRE].

[41] H.B. Nielsen and M. Ninomiya, No Go Theorem for Regularizing Chiral Fermions, Phys. Lett. B 105 (1981) 219 [INSPIRE].

[42] H.B. Nielsen and M. Ninomiya, Intuitive understanding of anomalies: A Paradox with regularization, Int. J. Mod. Phys. A 6 (1991) 2913 [INSPIRE].

[43] H. Kleinert, On the hadronization of quark theories, in Understanding the fundamental constituents of matter, A. Zichichi ed., Plenum Press, New York U.S.A. (1978).

[44] C. Itzykson and J-B. Zuber, Quantum Feild Theory, pg. 161, McGraw-Hill, Inc. ISBN 0-07-032071-3.

[45] T.P. Cheng and L.F. Li, Gauge theory of elementary particle physics, Oxford University Press Inc., New York, U.S.A. (1984), ISBN 978-019-851961-4.

[46] R.N. Mohapatra and P.B. Pal, Massive Neutrinos in Physics and Astro-physics, third edition, World Scientific, (2004).

[47] S.-S. Xue, Neutrino masses and mixings, Mod. Phys. Lett. A 14 (1999) 2701 [hep-ph/9706301] [INSPIRE].

[48] E. Eichten and J. Preskill, Chiral Gauge Theories on the Lattice, Nucl. Phys. B 268 (1986) 179 [INSPIRE].

[49] M. Creutz, M. Tytgat, C. Rebbi and S.-S. Xue, Lattice formulation of the standard model, Phys. Lett. B 402 (1997) 341 [hep-lat/9612017] [INSPIRE]. 
[50] S.-S. Xue, Why is the top quark much heavier than other fermions?, Phys. Lett. B 721 (2013) 347 [arXiv:1301.4254] [InSPIRE].

[51] S.-S. Xue, Higgs Boson and Top-Quark Masses and Parity-Symmetry Restoration, Phys. Lett. B 727 (2013) 308 [arXiv: 1308.6486] [InSPIRE].

[52] ATLAS collaboration, Search for contact interactions and large extra dimensions in dilepton events from pp collisions at $\sqrt{s}=7 \mathrm{TeV}$ with the ATLAS detector, Phys. Rev. D 87 (2013) 015010 [arXiv:1211.1150] [INSPIRE].

[53] D. Bai, J.-W. Cui and Y.-L. Wu, Quantum Electroweak Symmetry Breaking Through Loop Quadratic Contributions, Phys. Lett. B 746 (2015) 379 [arXiv:1412.3562] [InSPIRE].

[54] M. Gell-Mann and M. Lèvy, The axial vector current in beta decay, Nuovo Cim. 16 (1960) 705 [inSPIRE].

[55] J. Zinn-Justin, Quantum Field Theory and Critical Phenomena, Oxford University Press, (2002).

[56] J.L. Cardy, Scaling and the Renormalization Group in Statistical Physics, Cambridge University Press, (1997).

[57] E. Brézin and J. Zinn-Justin, Renormalization of the nonlinear $\sigma$-model in $2+\epsilon$ dimensions - Application to the Heisenberg ferromagnets, Phys. Rev. Lett. 36 (1976) 691 [INSPIRE].

[58] H. Kleinert, Particles and Quantum Field, World Scientific Publishing Company, (2016).

[59] S. Weinberg, Elementary particle theory of composite particles, Phys. Rev. 130 (1963) 776 [INSPIRE].

[60] S. Weinberg, Quasiparticles and the Born Series, Phys. Rev. 131 (1963) 440 [INSPIRE].

[61] S. Weinberg, Systematic Solution of Multiparticle Scattering Problems, Phys. Rev. 133 (1964) B232 [InSPIRE].

[62] S. Weinberg, Evidence That the Deuteron Is Not an Elementary Particle, Phys. Rev. 137 (1965) B672 [INSPIRE].

[63] S. Weinberg, Critical Phenomena for Field Theorists, in Understanding the Fundamental Constituents of Matter, in Understanding the Fundamental Constituents of Matter,

A. Zichichi ed., Plenum Press, New York, U.S.A. (1977).

[64] H. Kleinert, Particles and Quantum Field, Chapter 30, World Scientific Publishing Company, (2016), http://klnrt.de/b6.

[65] J. Preskill, Gauge anomalies in an effective field theory, Annals Phys. 210 (1991) 323 [INSPIRE].

[66] M.E. Peskin and D.V. Schroeder, An Introduction to Quantum Field Theory, Westview Press, (1995), ISBN-13 978-0-201-50397-5.

[67] Particle Data Group collaboration, J. Beringer et al., Review of Particle Physics (RPP), Phys. Rev. D 86 (2012) 010001 [inSPIRE].

[68] CMS collaboration, Search for massive WH resonances decaying to $\ell \nu \mathrm{b} \overline{\mathrm{b}}$ final state in the boosted regime at $\sqrt{s}=8 \mathrm{TeV}$, CMS-PAS-EXO-14-010 (2014).

[69] ATLAS collaboration, Search for high-mass diboson resonances with boson-tagged jets in proton-proton collisions at $\sqrt{s}=8 \mathrm{TeV}$ with the ATLAS detector, JHEP 12 (2015) 055 [arXiv: 1506.00962] [INSPIRE]. 
[70] CMS collaboration, Search for resonances and quantum black holes using dijet mass spectra in proton-proton collisions at $\sqrt{s}=8$ TeV, Phys. Rev. D 91 (2015) 052009 [arXiv: 1501.04198] [INSPIRE].

[71] CMS collaboration, Search for massive resonances in dijet systems containing jets tagged as $W$ or $Z$ boson decays in pp collisions at $\sqrt{s}=8$ TeV, JHEP 08 (2014) 173 [arXiv: 1405.1994] [INSPIRE].

[72] ATLAS collaboration, Search for Dark Matter in Events with Missing Transverse Momentum and a Higgs Boson Decaying to Two Photons in pp Collisions at $\sqrt{s}=8$ TeV with the ATLAS Detector, Phys. Rev. Lett. 115 (2015) 131801 [arXiv:1506.01081] [INSPIRE].

[73] CMS collaboration, Search for the production of dark matter in association with top-quark pairs in the single-lepton final state in proton-proton collisions at $\sqrt{s}=8 \mathrm{TeV}$, JHEP 06 (2015) 121 [arXiv: 1504.03198] [INSPIRE].

[74] J. Chang et al., An excess of cosmic ray electrons at energies of 300-800 GeV, Nature 456 (2008) 362 [INSPIRE].

[75] Y.-Z. Fan, B. Zhang and J. Chang, $e^{ \pm}$Excesses in the Cosmic Ray Spectrum and Possible Interpretations, Int. J. Mod. Phys. D 19 (2010) 2011 [arXiv:1008.4646] [InSPIRE].

[76] N. Arkani-Hamed, D.P. Finkbeiner, T.R. Slatyer and N. Weiner, A Theory of Dark Matter, Phys. Rev. D 79 (2009) 015014 [arXiv:0810.0713] [InSPIRE].

[77] D. Malyshev, I. Cholis and J. Gelfand, Pulsars versus Dark Matter Interpretation of ATIC/PAMELA, Phys. Rev. D 80 (2009) 063005 [arXiv:0903.1310] [InSPIRE].

[78] AMS collaboration, M. Aguilar et al., Precision Measurement of the Proton Flux in Primary Cosmic Rays from Rigidity 1 GV to $1.8 \mathrm{TV}$ with the Alpha Magnetic Spectrometer on the International Space Station, Phys. Rev. Lett. 114 (2015) 171103 [INSPIRE].

[79] http://pandax.physics.sjtu.edu.cn/.

[80] https://icecube.wisc.edu/.

[81] H. Kleinert and S.-S. Xue, Fermion Bound States in a Strongly-Coupled Fermion Liquid, in preparation. 